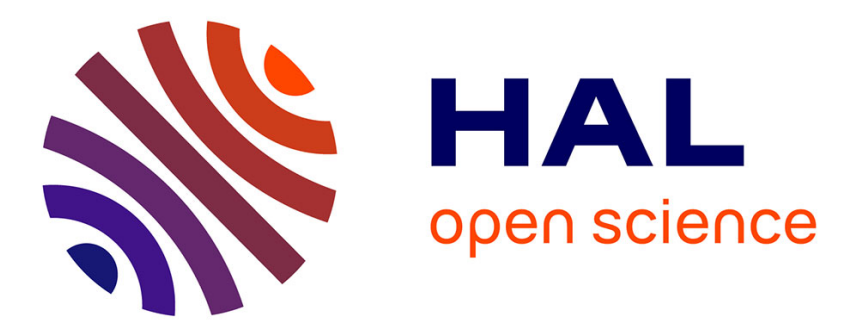

\title{
Experimental and numerical verification of bifurcations and chaos in cam-follower impacting systems
}

Ricardo Alzate, Mario Di Bernardo, Umberto Montanaro, Stefania Santini

\section{To cite this version:}

Ricardo Alzate, Mario Di Bernardo, Umberto Montanaro, Stefania Santini. Experimental and numerical verification of bifurcations and chaos in cam-follower impacting systems. Journal of Computational and Nonlinear Dynamics, 2007, 10.1007/s11071-006-9188-8 . hal-01304369

\section{HAL Id: hal-01304369 \\ https://hal.science/hal-01304369}

Submitted on 19 Apr 2016

HAL is a multi-disciplinary open access archive for the deposit and dissemination of scientific research documents, whether they are published or not. The documents may come from teaching and research institutions in France or abroad, or from public or private research centers.
L'archive ouverte pluridisciplinaire HAL, est destinée au dépôt et à la diffusion de documents scientifiques de niveau recherche, publiés ou non, émanant des établissements d'enseignement et de recherche français ou étrangers, des laboratoires publics ou privés.

\section{다(1)(2)}

Distributed under a Creative Commons Attribution - ShareAlikel 4.0 International 


\title{
Experimental and numerical verification of bifurcations and chaos in cam-follower impacting systems
}

\author{
R. Alzate - M. di Bernardo $\cdot$ U. Montanaro $\cdot$ S. Santini
}

\begin{abstract}
In this paper, we present the design, modelling and experimental validation of a novel experimental cam-follower rig for the analysis of bifurcations and chaos in piecewise-smooth dynamical systems with impacts. Experimental results are presented for a cam-follower system characterized by a radial cam and a flat-faced follower. Under variation of the cam rotational speed, the follower is observed to detach from the cam and then show the emergence of periodic impacting behaviour characterized by many impacts and chattering. Further variations of the cam speed cause the sudden transition to seemingly aperiodic behaviour. These results are compared with the numerical simulation of a mathematical model of the system which shows the same qualitative behaviour. Excellent quantitative agreement is found between the numerical and experimental results.
\end{abstract}

Keywords Piecewise-smooth dynamical systems . Impacting systems $\cdot$ Cam-follower $\cdot$ Bifurcations

\section{Introduction}

The analysis of complex behaviour in mechanical devices with impacts and other types of piecewise-smooth systems has been the subject of much on-going research (for example, see [9, 18, 33] and references therein). It has been shown that such systems can exhibit a novel class of non-linear phenomena, termed as discontinuity-induced bifurcations (DIBs). These events occur whenever one of the system invariant sets interacts nontrivially with the phase space boundaries where the system is discontinuous. DIBs can lead to dramatic changes of the system qualitative behaviour. It has been shown, for instance, that the transitions to chaos often observed in mechanical systems with impacts are due to grazing bifurcations of limit cycles [34]. These phenomena have been studied in detail and a consistent theory for their classification has been derived by a number of authors (e.g. see $[19,31]$ and references therein).

As the analysis of bifurcations in piecewise-smooth systems is further expanded, it is becoming increasingly important to carry out an extensive experimental investigation and validation of the theoretical results obtained. Complex behaviour in impacting systems has been observed experimentally in a number of papers in the literature. Examples include the early work on impact oscillators in [3, 5, 27, 37, 46, 48]. More recent papers include the work by Wiercigroch et al. reported in [52] and the results of Piiroinen et al. on impacting pendula [39]. (For further references see 
also the books $[7,51]$ and references therein). Particularly, cumbersome dynamics can be observed in the case of impact oscillators with moving boundaries. For example, in [11], it is suggested that a novel bifurcation phenomenon termed as corner-impact can occur in discontinuously forced impact oscillators.

Cam-follower devices are an important class of impacting systems which are indeed used in a wide range of applications. Typically, the rotation of the cam at some constant speed provides the forcing to operate the follower. The most common example is that of valve trains of internal combustion engines, where the cam rotation imparts through the follower the proper motion to the engine valves while a spring provides the restoring force necessary to maintain contact between the components [25].

Typically, the cam is designed to rotate at a constant velocity. In practice, often such velocity is varied either by unwanted fluctuations and noise or to satisfy certain design specifications. For instance, in a valve train the cam-follower mechanism works at speeds ranging from about $2500 \mathrm{rpm}$ in large automobile engines to over $10000 \mathrm{rpm}$ in motorcycle racing engines. Despite the design effort, as the engine speed increases, the follower motion can be substantially different from the desired kinematic behaviour [40]. In particular, above a critical rotational speed, the follower detaches from the cam and then impacts intermittently with it (causing the well-known valve floating phenomenon in automotive applications) [35].

It has been observed that, under variations of the cam rotational speed and other parameters, the follower can exhibit complex behaviour including bifurcations and chaos [53, 54]. Despite their importance in applications, the investigation of these phenomena has been studied little in the existing literature and mostly in the context of smooth non-linear dynamics. Thus, little is known of the nature of the bifurcation phenomena leading to the complex behaviour which is often observed after the follower detachment from the cam.

This paper is concerned with the design, modelling and investigation of a novel experimental rig aimed at testing and analyzing the behaviour of impacting systems and in particular cam-follower systems. Specifically, the aim is to gain an understanding of the complex dynamics associated to the impacting motion following the detachment of the follower from the actuating cam. The aim of the experiment is twofold: (i) to observe and classify the bifurcation phenomena detected in the system; (ii) to provide a new, versatile test rig to validate the latest theoretical results on impacting mechanical systems. In this context, cam-follower based systems can be chosen as a general and useful benchmark problem as they are widely used in various machines and mechanical engineering devices [35].

Also, we wish to point out some practical issues that further motivate our research (although an exhaustive discussion on all the implications deriving from the use of novel non-linear analysis tools within the context of cam-follower system design is out of the scope of this work).

- In high-speed cams, the presence of unavoidable camshaft fluctuations can affect the accuracy of the follower motion [16, 53]. Starting from the pioneering work of Rothbart [41], engineers have highlighted the potential advantages of using variable-speed cams and embedding their variablespeed explicitly as a parameter into the design process. We wish to emphasize that the experimental investigation of the non-linear dynamics of the follower after its detachment from the cam can be used to improve the cam design. For example, bifurcation diagrams can be obtained to evaluate the influence on the cam-follower dynamics of various parameters including the cam rotational speed, variable stiffness characteristics, different cam profiles, etc [54].

- The occurrence of impacts is particularly undesired in those applications that require to operate the cam at very variable velocities. Here, as mentioned above, the most common and significant example is the valve train of an internal combustion engine where the occurrence of a contact loss between the cam and the follower at a high speed revolution $[29,47]$ causes a reduction in the engine performance (due to valve bouncing and floating) in terms of engine efficiency, fuel consumption and emissions [15]. It has been experimentally observed, that at a certain engine speed termed limit speed, the valve bounce amplitude increases dramatically, thereby resulting in what seems to be a chaotic valve motion [35]. Although the occurrence of impacts sets narrow bounds onto the maximal engine velocity, there is a generic trend to advance the limit speed in both passenger and racing automobiles and/or motorbikes. Increasing the engine limit speed allows the engine to run faster and in turn to produce more power. In addition, operating at higher engine speed is also strongly desirable since 
it is possible to design smaller and lighter engines that produce the same power as larger, heavy engines. Therefore, the reduction of valve bouncing and/or valve floating, as the velocity increases, is established as a primary goal of valve design based on the camfollower mechanism $[15,32]$. We remark that in current engines, a prefixed and sufficiently large spring force (and pre-load) is always applied to the camfollower joint to keep the contact throughout the entire rotation [40]. As a natural consequence, there is an increase in the contact force, which induces higher stresses possibly leading to early surface failure of the parts. Moreover, the high-friction valve train reduces the efficacy of the engine system, that works harder to push the follower through its motions [49].

Understanding the complex dynamics of these systems can then be relevant in those applications where it is essential to avoid unwanted impacting behaviour. Indeed, a deeper insight of the postdetachment dynamics could unveil less conservative solutions for detachment avoidance. For example, bifurcation control techniques or active controllers could be used without requiring the use of a stiff closing spring or the design of much more complex desmodromic valves [12]. Also, an exhaustive study on the occurrence of gaps between connecting components will also allow a better understanding of the nature of the resulting noise, vibration, wear and mechanical stress often observed in applications.
The rest of the paper is outlined as follows. In Section 2, the experimental rig is presented together with its implementation. Then, experimental runs are reported in Section 3, showing the main types of dynamical behaviour exhibited by the system. A detailed model of the rig is derived in Section 4, while parameter identification is carried out in Section 5. Here, the comparison between the experimental and numerical results is presented, showing excellent agreement between the expected and observed dynamics.

\section{The experimental rig}

\subsection{Description}

The experimental rig considered in this paper is shown in Fig. 1. As mentioned above, the aim of the rig is to investigate the dynamics of a cam-follower system with particular attention to the impacting behaviour following the detachment of the follower from the cam. In contrast to what presented in [53], our rig is based on a radial cam with an oscillating flat-faced follower. This type of cam-follower systems are increasingly used in automotive industry in the actuation of overhead valve trains [35].

Notice that the small follower mass and its high rotational speed do not allow feasible and accurate contact measures (by means of piezoelectric transducers).

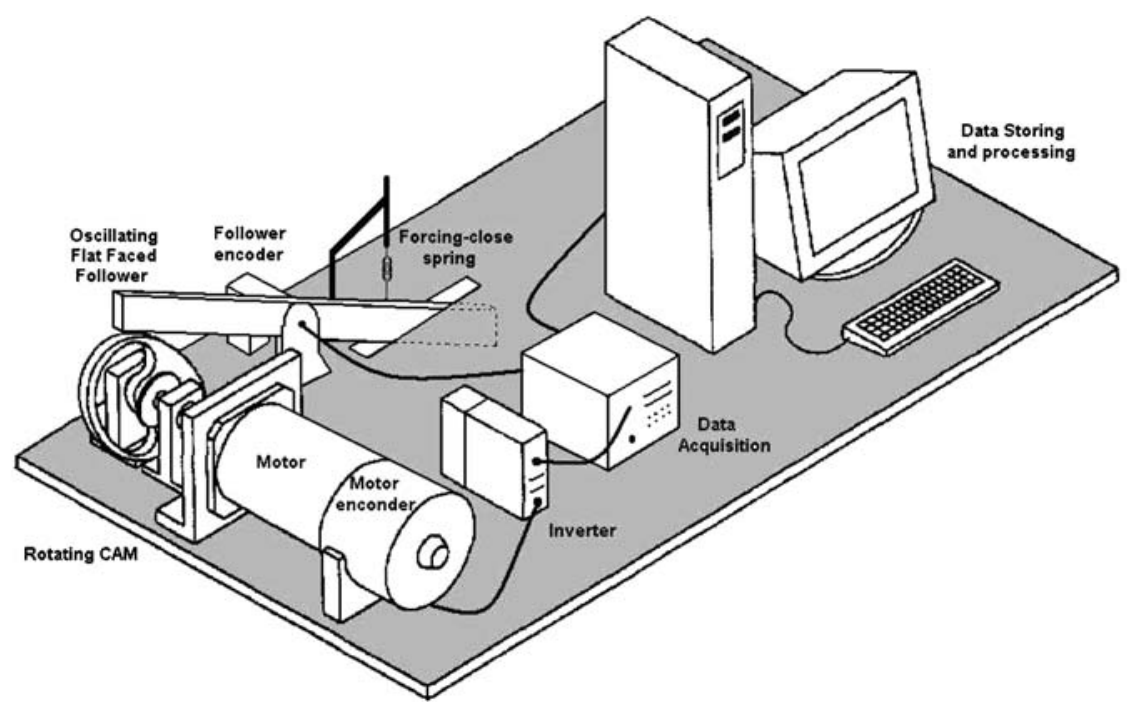

Fig. 1 Schematic of the experimental rig 
Moreover, the high frequency range makes it unfeasible to use proximity transducers. Therefore, a different geometry (where for example the cam rotation results in a linear motion imparted to the follower) would make the accurate measurement of the relative motion between the cam and the follower more cumbersome and possible only by sophisticated optical laser transducers. In general, such transducers are expensive and their use implies several constraints on the design.

The rotational geometry of the rig proposed here makes it easier to measure the follower motion and the cam position directly from the cam-shaft and followershaft rotational angles. Furthermore, the choice of an oscillating arm gives a great advantage in terms of reduction of friction at contact points and consequently wearing of pieces and ease of replacement [35]. Also, from a non-linear dynamics viewpoint, the rotational nature of the follower motion makes it easier to avoid the presence of unwanted stick-slip motion due to friction which would make the theoretical understanding of the phenomena observed particularly cumbersome.

These considerations informed the design of the experimental apparatus shown in Fig. 1 where the cam pushes a rotational follower attached through a spring to a rigid fixed iron frame. Note that the cam-follower system in the rig can be assumed to be stiff and large enough to reduce possible vibrations induced by the inertial force. The cam is also equipped with a flywheel which is designed ad hoc in order to compensate undesired oscillations and torque variations due, for example, to the occurrence of impacts. Additional details on the material and the dimensions of each individual components of the rig are given in Table 1.

Table 1 Details on materials and dimensions

\begin{tabular}{lc}
\hline Part & Description \\
\hline $\begin{array}{l}\text { Cams, flywheels and } \\
\text { follower contact surface }\end{array}$ & $\begin{array}{c}\text { Made of low-alloy hardened } \\
\text { stainless steel } \\
\text { UNI38NiCrMo4 }\end{array}$ \\
Follower body & Made of aluminium (Al) \\
Cam internal radius & $30 \mathrm{~mm}$ \\
Cam external radius & $60 \mathrm{~mm}$ \\
Cam eccentricity & $15 \mathrm{~mm}$ \\
Flywheel radius & $80 \mathrm{~mm}$ \\
Follower length & $600 \mathrm{~mm}$ \\
Follower width & $16 \mathrm{~mm}$ \\
Follower height & $60 \mathrm{~mm}$ \\
\hline
\end{tabular}

\subsection{The cam profile}

An important aspect of cam-follower systems is the cam profile. This is typically designed in order to provide the forcing to the follower which is required for it to operate in some desired manner. Usually, the cam profile is obtained by solving a constrained optimization problem (see [35] for further details). Given the wide range of applications, there is a wide variety of possible cam geometries ranging from cycloidal cams to those designed using splines that can even provide discontinuous acceleration to the follower [36]. For this reason, the experimental rig was designed in order to allow easy and direct access to the cam and the flywheel for their possible replacement. Currently, two different types of cam can be alternatively mounted on the experiment which provide, respectively, a simple harmonic motion (eccentric circular cam) and a profile characterized by discontinuities in the acceleration. In this work, we will show experiments related to an eccentric circular cam, which are often used to produce motion in pumps or to operate steam engine valves [35]. Other examples of various applications based on the eccentric circular cam can be found in [10, 14, 20, 22, 50]. The use of circular cams in the automotive field is instead reported in $[13,44]$. On-going research activity is dealing with evaluating the effects on the follower motion of a discontinuity in the forcing [38].

It is worth mentioning here that by appropriately designing the cam profile it would be possible to impart any type of desired motion to the follower. This makes the experiment described in this paper an extremely versatile and flexible tool to investigate the non-linear dynamics of impacting mechanical systems.

\subsection{Implementation}

The physical implementation of the experimental rig described above is depicted in Fig. 2(a)-(b), where the mechanical device is shown to be appropriately coupled to electronic systems for the acquisition, storage and processing of experimental data. The main feature of the experimental set-up can be summarized as follows:

- The cam motion is controlled by a brushless motor driven through an embedded controller. Notice that the angular position and velocity of the cam and the driving motor are assumed to be identical because 
of a rigid coupling connecting the cam to the motor shaft.

- The measures of the cam and the follower angular position are obtained through high-resolution optical encoders mounted respectively on the cam and follower shaft.

- Reliable AD/DA conversions and signal processing are implemented through DSPACE [24], a widely used commercial data acquisition integrated hardware-software system (16 bit, $250 \mathrm{MHz}, \mathrm{PCI}$ interface).

- The signals are processed and analyzed using MATLAB [26].

Additional details are included in Table 2. (A more extensive description of the experimental rig can be found in [17].)
Table 2 Additional details on the instrumentation

\begin{tabular}{ll}
\hline Device & Description \\
\hline Servo (motor-driver) system & Sanyo-Denki Q-series [42] \\
Data acquisition system & dSPACE ACE-kit \\
& ACE1104CLP [24] \\
Follower position encoder & HENGSTLER RI-58 D [23] \\
Cam position resolution & 5000 pulses per revolution \\
Follower position resolution & 10000 pulses per revolution
\end{tabular}

\section{Experimental results}

As reported in the literature (for example, see [35]), cam-follower systems can be particularly sensitive to variations of the cam rotational speed, $\omega$. Here, we shall seek to uncover the complex dynamics observed experimentally under variations of $\omega$ due to the occurrence of collisions between the cam and the follower.

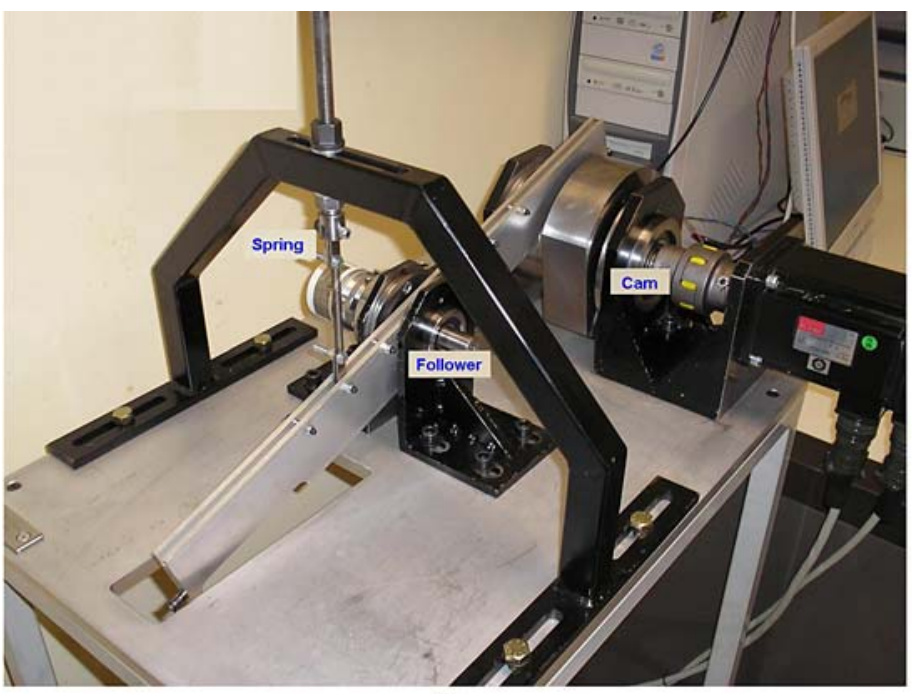

(a)

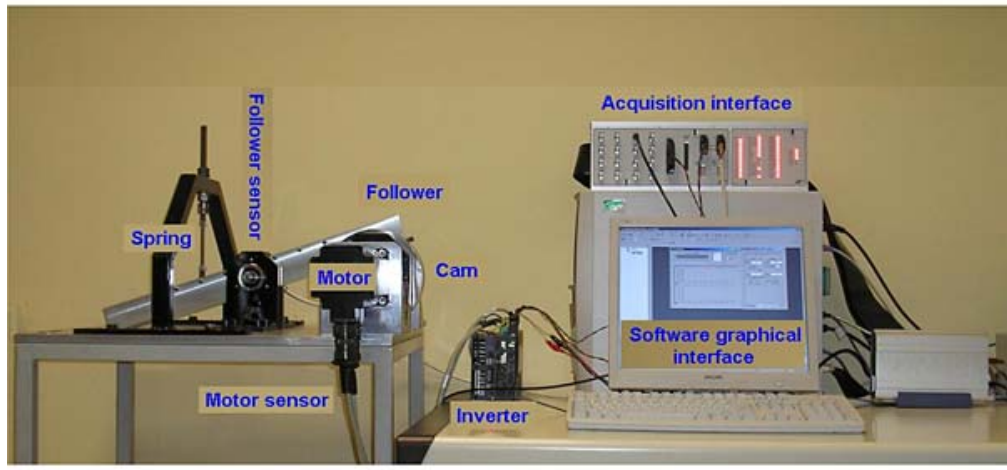

(b)

Fig. 2 Pictures of the experimental rig: (a) view from above; (b) front view 
In order to visualize the experimental results, the first step is to acquire the cam profile as described below.

\subsection{Acquiring the cam profile}

The exact cam profile that drives the follower motion can be derived by an automated numerical calculation that returns the cam profile as a function of its experimentally measured angular position.

The identification has been carried out by using the curve fitting toolbox available in MATLAB and working with a subset of data corresponding to one revolution of the cam at low steady-state values of its velocity. This condition obviously ensures the continuous contact between the cam and the follower. The estimation procedure gives the angular displacement, $\hat{\theta}_{c}$, at the follower joint due to the rotation of the cam when the cam and the follower are in contact, as a function of the cam angular position, $\theta_{c}$. In particular, a seventh-order Gaussian model was used of the form

$\hat{\theta_{c}}:=f\left(\theta_{c}\right)=\sum_{i=1}^{7} a_{i} e^{-\left(\frac{\theta_{c}-b_{i}}{c_{i}}\right)^{2}}$

where $\theta_{c}$ and $\hat{\theta}_{c}$ are expressed in radians, and the coefficients $a_{i}, b_{i}$ and $c_{i}$ are estimated numerically using a least-squares algorithm as reported in Table 3.

This result was also validated by deriving a theoretical estimate of the cam profile based on a geometric approach (see Appendix B). Despite its validity, this latter approach relies on the exact knowledge of the cam profile that is typically available if the cam profile is simple enough. In general, we found the practical methodology described above can be more easily adapted to different cam profiles.

A typical experimental time series obtained using such a methodology is shown in Fig. 3. Here, we depict the time evolution of the estimated angular displace-

Table 3 Estimates of coefficients in (1)

\begin{tabular}{llcl}
\hline$i$ value & $a_{i}$ & \multicolumn{1}{l}{$b_{i}$} & $c_{i}$ \\
\hline 1 & 0.307 & 6.311 & 2.777 \\
2 & 0 & -0.19 & 0.349 \\
3 & 0.304 & -0.753 & 2.613 \\
4 & 0.013 & 5.208 & 1.268 \\
5 & 0 & 4.339 & 0.067 \\
6 & 0.095 & 1.932 & 2.251 \\
7 & 0.036 & 4.418 & 1.743 \\
\hline
\end{tabular}

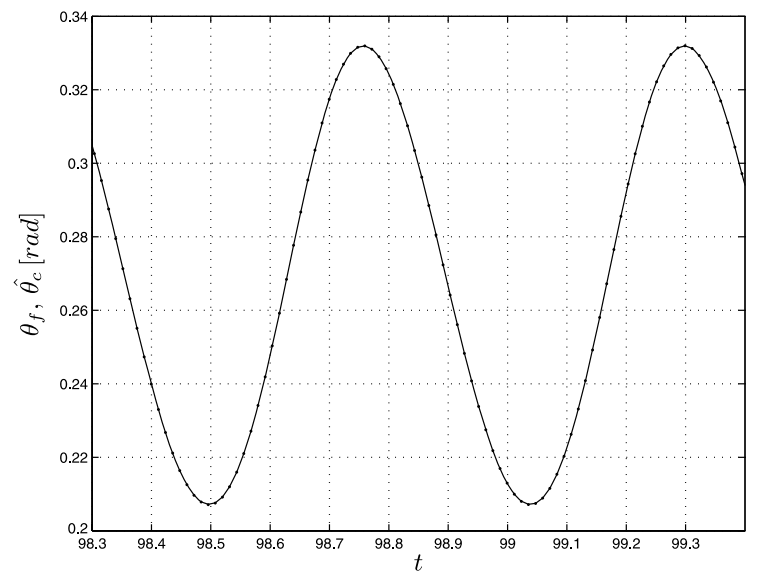

Fig. 3 Time evolution of $\hat{\theta_{c}}$ (dotted line) and $\theta_{f}$ (solid line) for $\omega=110 \mathrm{rpm}$. We observe that the two signals are perfectly overlapping in practice. See Fig. 4 for an illustration of the related error signal

ment $\hat{\theta}_{c}$ and the measured follower angular position $\theta_{f}$ when the follower and the cam are in permanent contact.

\subsection{Experimental time series}

Using the experimental rig and the acquisition methodology described above, it is now possible to uncover the complexity of the dynamical behaviour exhibited by the system under parameter variations.

In general, we observe three regions of different qualitative behaviour associated to different values of $\omega$ :

- Permanent contact, where the cam and the follower stay in contact for all time.

- $P(m, n)$ impacting behaviour, where the follower exhibits periodic motion characterized by $m$ impacts per period; The period $T$ being equal to $2 \pi n / \omega$.

- Aperiodic motion and chaos.

We now give some representative examples of each of these types of behaviour.

\subsubsection{Permanent contact - low velocity regime}

In Fig. 3, the dynamics of the follower at a constant cam angular velocity $\omega=\dot{\theta}_{c}=110 \mathrm{rpm}$ is shown.

At this velocity, the restoring force of the follower is higher than the force exerted by the constraint represented by the cam. In this condition, the cam and the 
follower remain in contact for all time, hence we have $\theta_{f}=\hat{\theta}_{c}$.

It is important to note that permanent contact is experimentally detected up to approximately $125 \mathrm{rpm}$ of $\omega$. For this reason, the set of values $\omega<125 \mathrm{rpm}$ will be denoted as the low velocity region.

\subsubsection{Detachment}

Past the low velocity region, the detachment of the mechanical components is observed. Detachment occurs when the force exerted by the cam over the follower while in contact exceeds the restoring force. As a consequence, we observe temporary unconstrained follower motion. Experimentally, this phenomenon was first observed at $\omega=\omega_{d} \approx 125 \mathrm{rpm}$, as shown in Fig. 4, where the difference between the cam and the follower position is shown for values of $\omega$ immediately lower and higher than $\omega_{d}$. Clearly, as the cam speed increases we observe the emergence of peaks in the error dynamics associated to the temporary detachment of the follower from the cam and the ensuing impacting behaviour.

\subsubsection{Periodic solutions}

For values of $\omega>\omega_{d}$, we observe the follower motion to exhibit periodic impacting behaviour characterized by different number of impacts per period. Examples of such behaviour are depicted in Fig. 5 where the cam and follower positions are shown (left panel) together with their difference (right panel).

\subsubsection{Aperiodic solutions}

Past a critical value of the cam velocity, the follower starts exhibiting aperiodic behaviour and sensitive dependence on initial conditions. Representative cases are shown in Figs. 6 and 7 for values of $\omega>155 \mathrm{rpm}$.

\subsection{Experimental bifurcation diagram}

To gain an understanding of the transition from periodic to aperiodic motion, an experimental bifurcation diagram was derived by sampling the follower evolution synchronously with the cam forcing period. The experimental diagram is reported in Fig. 8. Examples
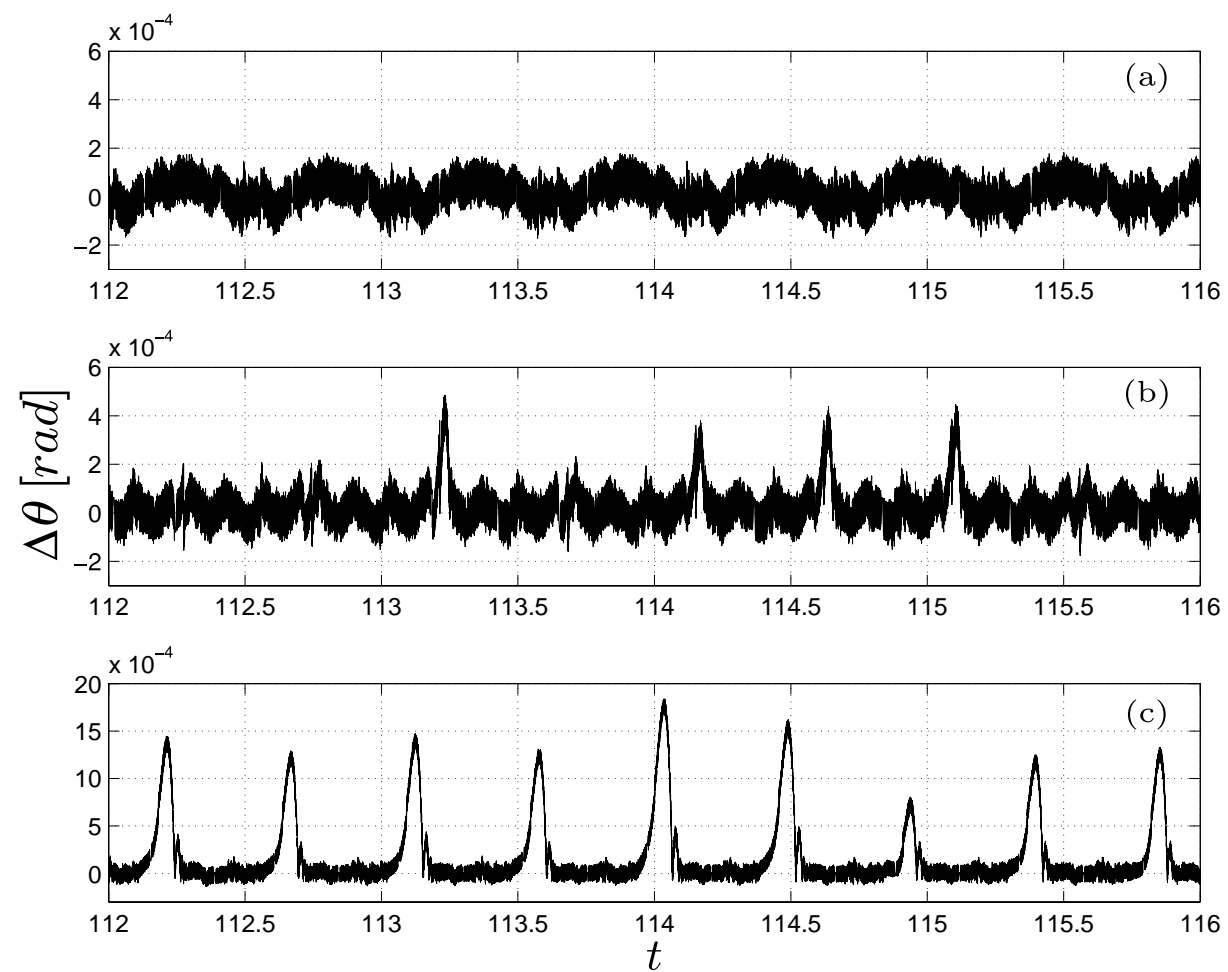

Fig. 4 Time evolution of the experimental difference between $\theta_{f}$ and $\hat{\theta_{c}}\left(\Delta \theta=\theta_{f}-\hat{\theta_{c}}\right)$ for (a) $\omega=110 \mathrm{rpm},(\mathrm{b}) \omega=125 \mathrm{rpm}$ and (c) $\omega=131 \mathrm{rpm}$ 


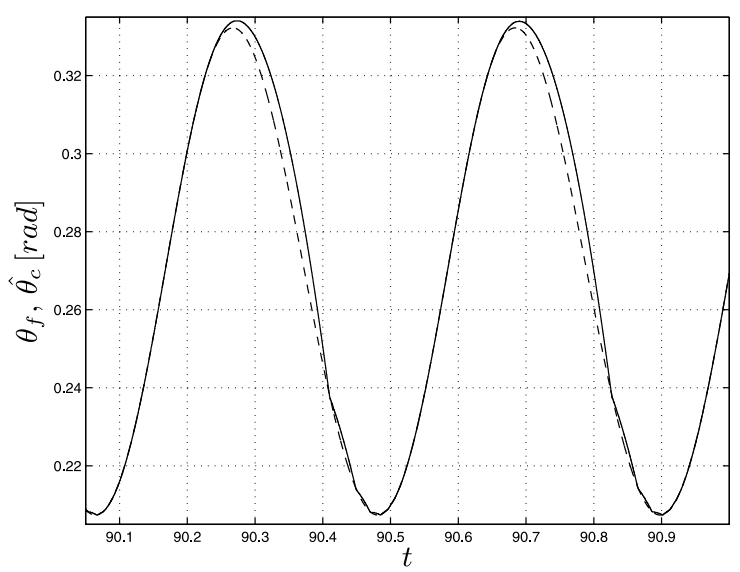

(a)

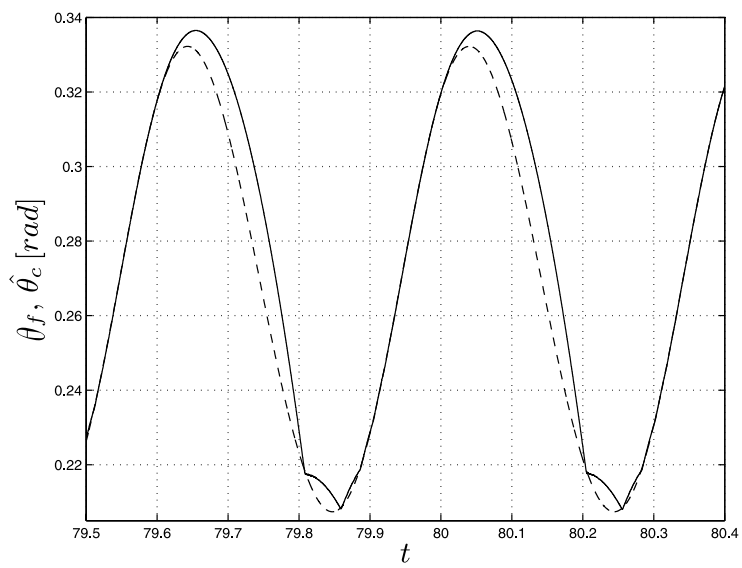

(c)

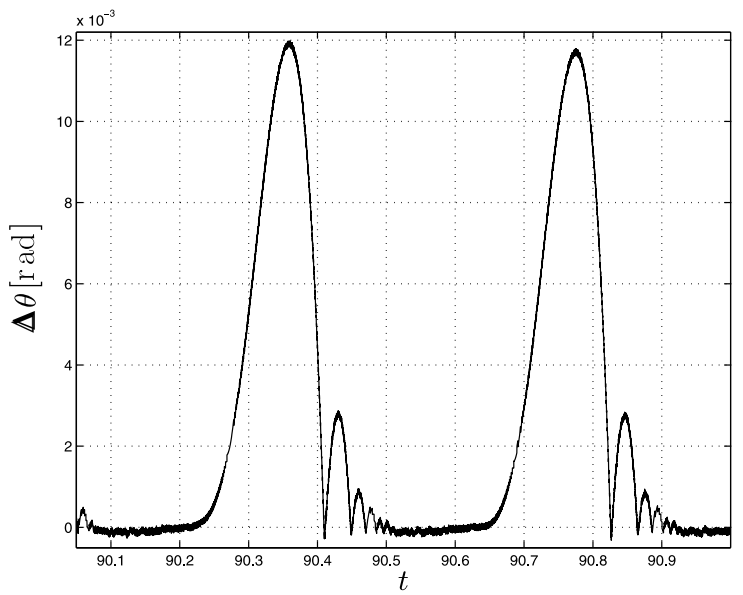

(b)

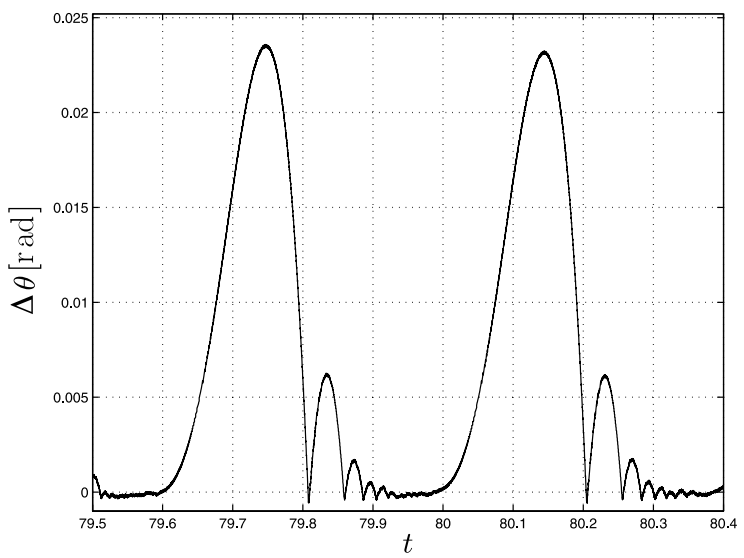

(d)

Fig. 5 Sections of experimental time series for the cam (dashed line) and follower (solid line) positions and their corresponding difference $\Delta \theta$ at (a), (b) $\omega=143 \mathrm{rpm}$ and (c), (d) $\omega=150 \mathrm{rpm}$
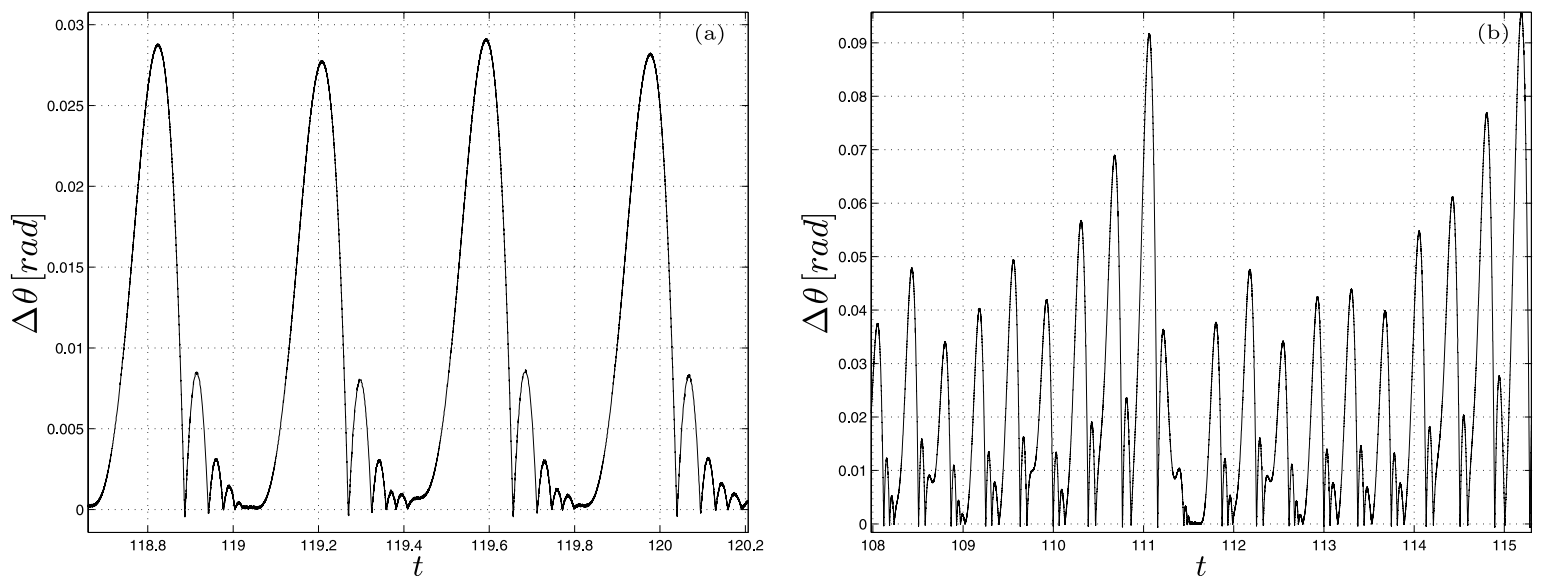

Fig. 6 Examples of (a) periodic and (b) chaotic evolution of the difference $\Delta \theta$ between $\theta_{f}$ and $\hat{\theta}_{c}$ for (a) $\omega=155 \mathrm{rpm}$ and (b) $\omega=159$ rpm 

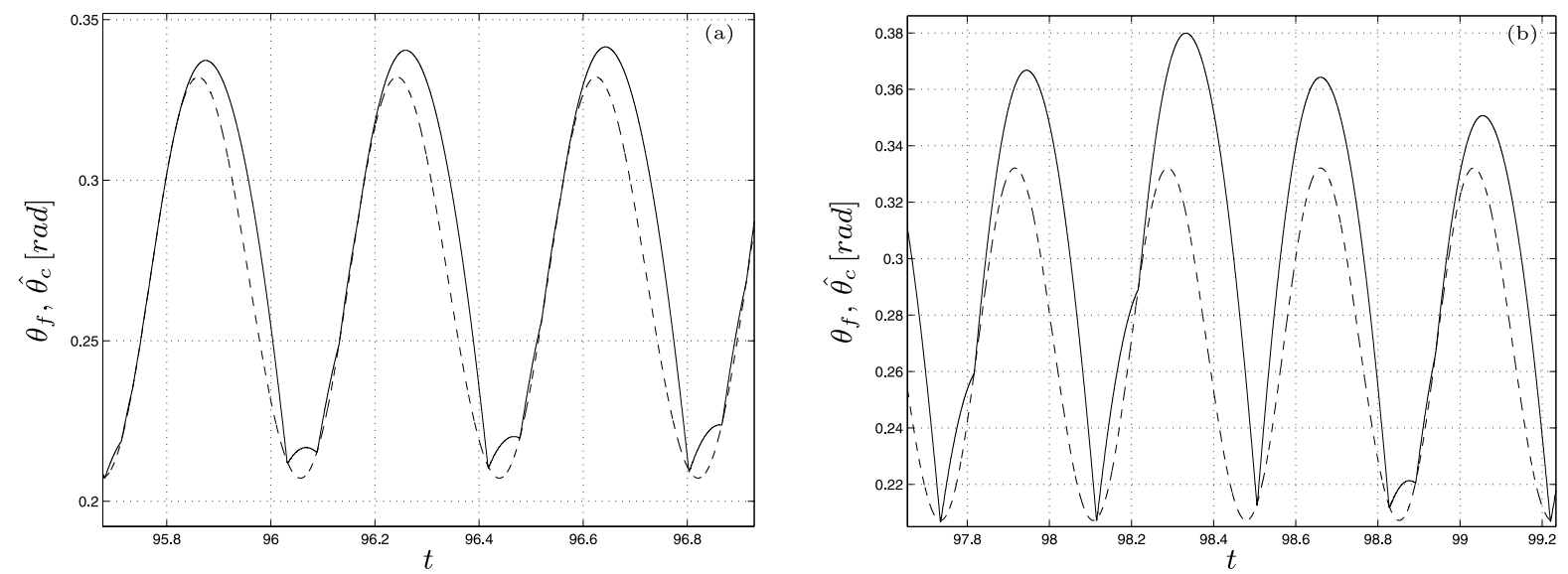

Fig. 7 Chaotic evolution of the follower position $\theta_{f}$ (solid line) at (a) $\omega=158 \mathrm{rpm}$ and (b) $\omega=160 \mathrm{rpm}$. The dashed line represent the profile $\hat{\theta_{c}}$

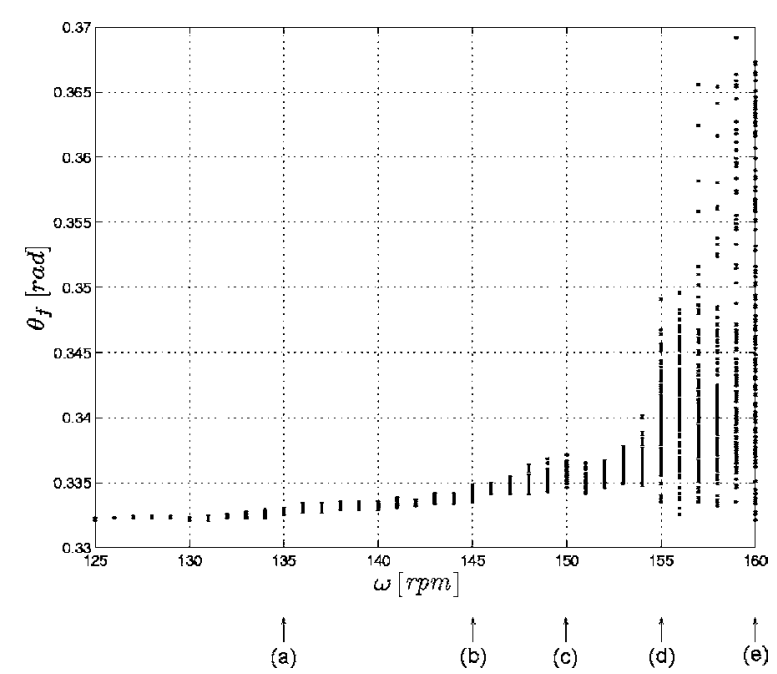

Fig. 8 Experimental bifurcation diagram of the follower position $\theta_{f}$ against the cam rotational speed $\omega$

of time trajectories at different points in the diagram are shown in Fig. 9.

The diagram shows that past the detachment point at about $125 \mathrm{rpm}$, the periodic behaviour of the system shares the same period of the cam forcing signal for a relatively large range of values of the cam speed $\omega$. Then, suddenly at about $155 \mathrm{rpm}$, the abrupt transition is observed to qualitative different behaviour which seems to be quickly evolving into a chaotic attractor (points $(d)$ and (e) in the diagram and in Fig. 9).

\section{Mathematical modelling}

In order to capture the qualitative behaviour of the system and validate the observed experimental behaviour, a proper mathematical model of the system is derived below. The formulation of an appropriate model can be a challenging task for most applications. In the case of cam-follower systems, various models have been proposed characterized by different degrees of complexity, ranging from simple models with 1 DOF (Degree of Freedom) [28] to complex models with 21 DOF [43] that use the additional DOF to include the effects of camshaft torsion and bending, backlash, squeeze of lubricant in bearings and so on. Nevertheless, there is general agreement, confirmed by experience in different applications, that a lumped parameter single degree of freedom model is adequate to represent most aspects of the dynamic behaviour of a cam-follower system (for example, see [2, 4, 21, 28]).

Rather than neglecting the presence of impacts, the cam-follower is regarded here as a single degree of freedom impacting oscillator with a unilateral constraint.

More precisely, as explained in [8], we model the follower dynamics under the external forcing $u(t) \in \mathbb{R}$ provided by the cam as

$$
\begin{aligned}
& \ddot{q}=g(q, \dot{q}, u), \\
& f(q, t) \geq 0,
\end{aligned}
$$




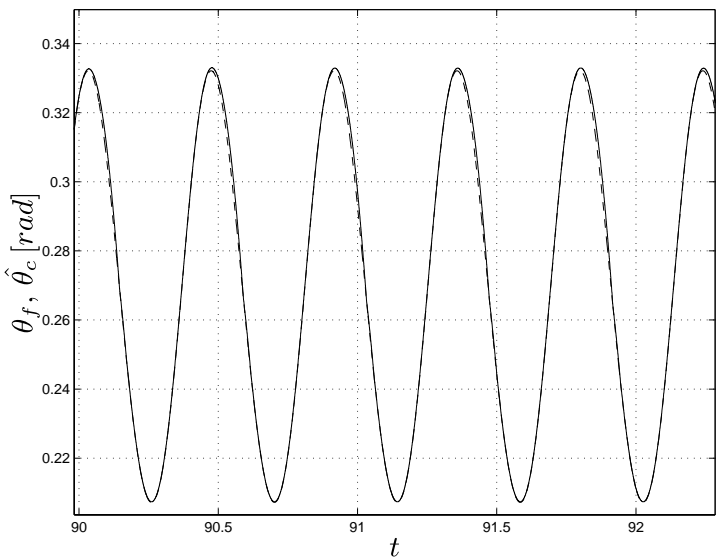

(a)

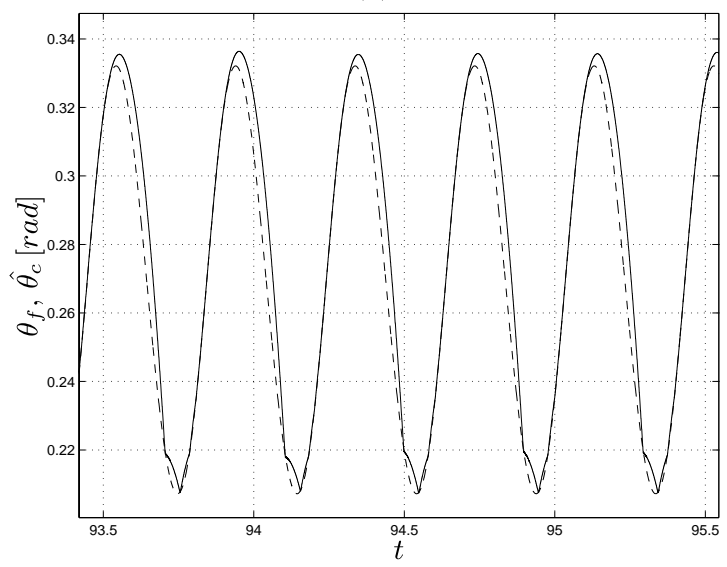

(c)

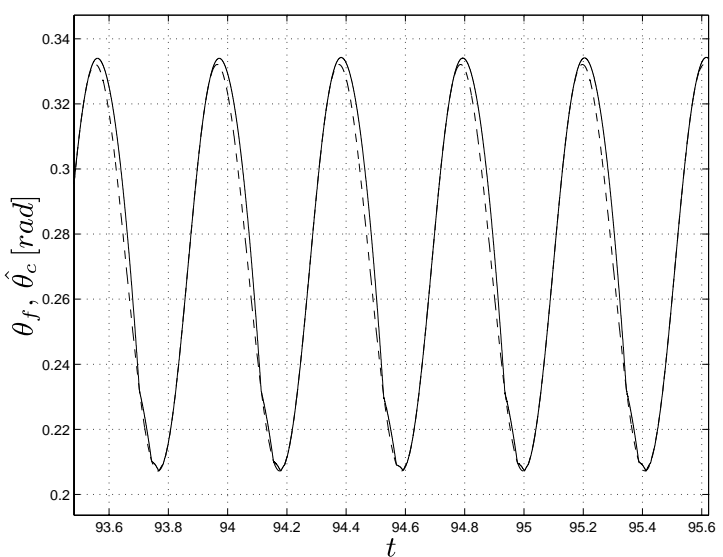

(b)

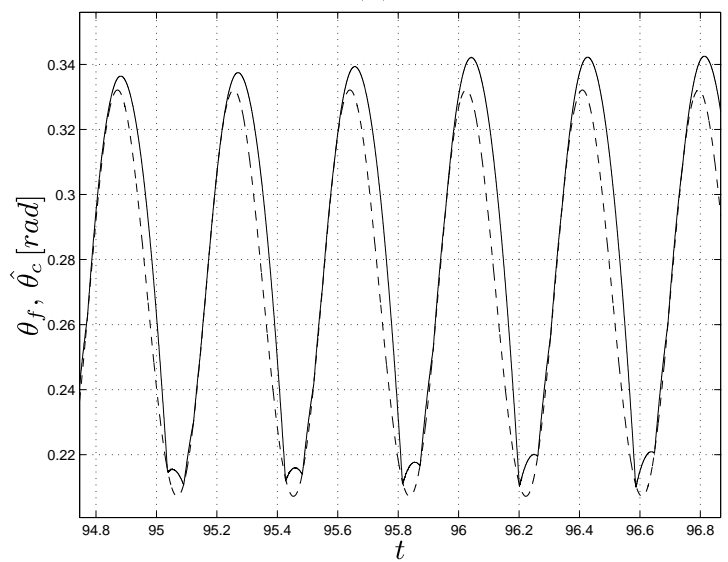

(d)

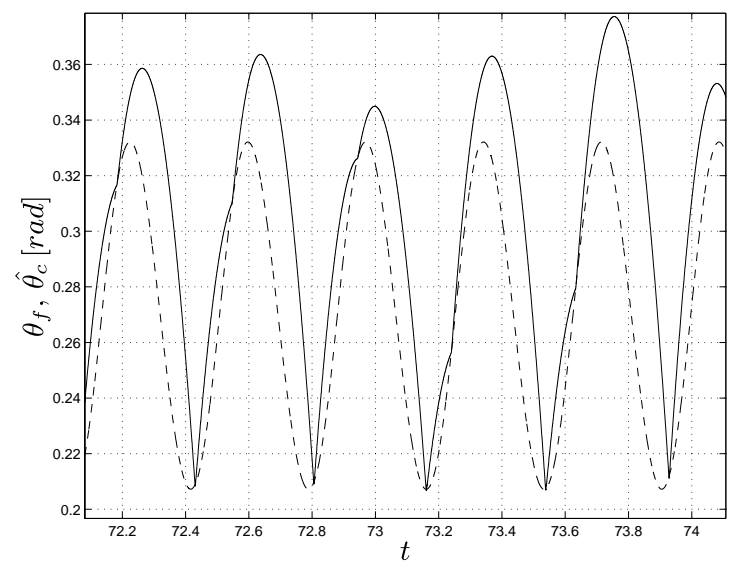

(e)

Fig. 9 Time evolution of the system at the values of $\omega$ labelled in Fig. 8. Specifically: (a) $\omega=135 \mathrm{rpm}$, (b) $\omega=145 \mathrm{rpm}$, (c) $\omega=150 \mathrm{rpm},(\mathrm{d}) \omega=155 \mathrm{rpm}$ and (e) $\omega=160 \mathrm{rpm}$. Solid lines represent follower position $\theta_{f}$ while dashed lines are related with the cam profile $\hat{\theta_{c}}$ 
Table 4 Summary of the general notation used in Section 4

\begin{tabular}{ll}
\hline Symbol & Description \\
\hline$\theta_{f}$ & Angular position of follower with counterclockwise sense of rotation \\
$\theta_{c}$ & Angular position of cam with counterclockwise sense of rotation \\
$\hat{\theta}_{c}$ & Angular displacement of the follower joint when in contact with the cam \\
$(\hat{t}, \hat{n})$ & Reference system attached to the follower \\
$(\tilde{x}, \tilde{y})$ & Reference system obtained by translating the axes $(x, y)$ to $\left(x_{0}, y_{0}\right)$ \\
$\left(\tilde{x}_{c}, \tilde{y}_{c}\right)$ & Reference system pivoted at the cam with origin $\left(x_{0}, y_{0}\right)$ \\
$p_{0}=\left(x_{0}, y_{0}\right)$ & Coordinates of the rotational center in the $(x, y)$ system \\
$p_{A}=\left(x_{A}, y_{A}\right)$ & Hooking point for spring \\
$p_{B}=\left(x_{B}, y_{B}\right), p_{P}=\left(x_{P}, y_{P}\right)$ & Ending points of the mechanical element that avoids rotation of spring \\
$p_{C}=\left(x_{c}, y_{c}\right)$ & Point on the cam surface which is nearest to $\Sigma$ \\
$p_{E}=\left(x_{E}, x_{E}\right)$ & Intersection between vertical line passing through $p_{A}$ and $g(x)=\tan \left(\theta_{f}\right) x$ \\
$p_{F}=\left(x_{f}, y_{f}\right)$ & Point of follower surface that will impact on the cam ${ }^{\mathrm{b}}$ \\
$p_{G}=\left(x_{g}, y_{g}\right)$ & Geometric center of the rotating cam \\
$d$ & Half height of follower \\
$d_{0}$ & Relaxed spring length \\
$d_{1}$ & Distance between $p_{B}$ and $p_{P}$ \\
$d_{2}$ & Distance between $p_{P}$ and $p_{E}$ \\
$e$ & Cam eccentricity \\
$K$ & Spring stiffness \\
$J$ & Follower moment of inertia \\
$\rho$ & Distance between the origin of the axis $(x, y)$ and $p_{E}$ \\
$\Sigma$ & Boundary of follower surface that becomes in contact with cam \\
$h$ & Distance between $p_{C}$ and $\Sigma^{\mathrm{c}}$ \\
$\bar{d}$ & Intersection between $\Sigma$ and the axis $y$ (it is equal to $\left.-d / \cos \left(\theta_{f}\right)\right)$ \\
\hline
\end{tabular}

\footnotetext{
${ }^{\mathrm{a}}$ Measured as the relative rotation of the coordinate system $\left(\tilde{x}_{c}, \tilde{y}_{c}\right)$ with respect to $(\tilde{x}, \tilde{y})$

${ }^{\mathrm{b}}$ This point is $(l,-d)$ in the coordinate system $(\hat{t}, \hat{n})$ and $\left(x_{f}, y_{f}\right)$ in the coordinate system $(x, y)$

${ }^{\mathrm{c}}$ It is straightforward that under contact $h$ is zero and $\Sigma$ is tangent to the cam at point $p_{C}$
}

where $q \in \mathbb{R}^{n}$, is the vector of the generalized coordinates of the follower system, $g$ is the system vector field and the real valued function $f$ represents the unilateral constraint on the follower position $q$. Note that $u(t)$ is nonzero only when the two bodies are in contact (i.e. when $f(q, t)=0)$. Using the terminology of complementarity systems, we say that the two variables $f$ and $u$ are complementary in the sense that $0 \leq u \perp f \geq 0$ (see [9] for further details).

The hybrid structure of model (2) allows to deal mathematically with the presence of intermittent contacts between the cam and the follower during the motion of the system. In particular, we can distinguish two different phases of motion as follows:

1. Unconstrained mode, when no contact occurs between the two bodies. From the modelling viewpoint, the follower dynamics simply reduces to an unforced harmonic oscillator $(f(q, t)>0$ and $u(t)=0)$;
2. Constrained mode, when permanent contact between the two bodies is established. Here the follower dynamics are induced by the specific cam profile $(f(q, t)=0$ and $u(t)>0) .^{1}$

In the following subsections, each particular mode of the follower motion will be modelled in accordance with both the schematic diagram depicted in Fig. 10 and the general notation reported in Table 4.

\subsection{Follower motion}

To derive a mathematical model of the follower motion, we use a standard Lagrangian approach. In particular, the system description can be obtained by solving the

\footnotetext{
${ }^{1}$ The follower motion is constrained to a phase space region bounded by the cam angular position.
} 


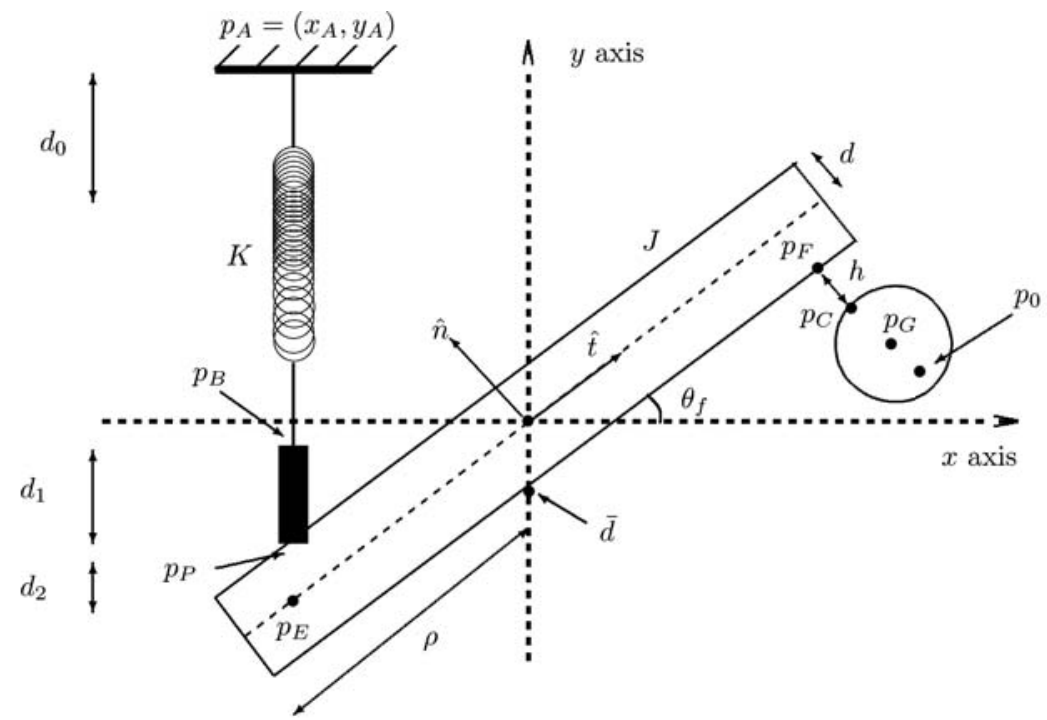

(a)

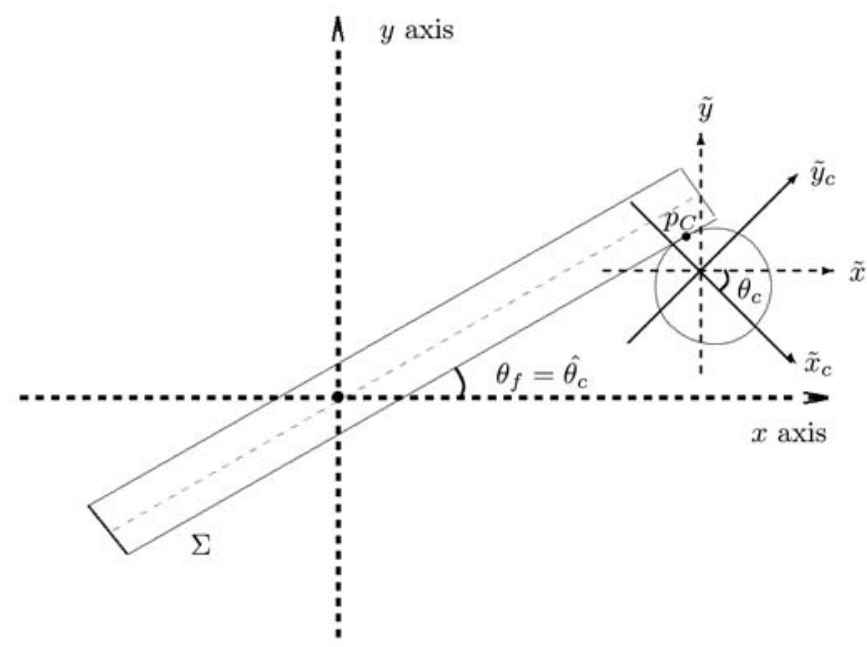

(b)

Fig. 10 Schematic diagram of the cam-follower system: (a) unconstrained mode; (b) constrained mode. All the labels are defined in Table 4

following equation

$\frac{\mathrm{d}}{\mathrm{d} t} \frac{\partial L}{\partial \dot{\theta}_{f}}-\frac{\partial L}{\partial \theta_{f}}=\tau$

where $L$ is the Lagrangian function, defined as the difference between the potential energy $(U)$ and the kinetic energy $(T)$ of the system

$L=T-U$, and $\tau$ is the external torque given by the nonconservative forces.

Let $f_{e}$ be the elastic force exhibited by the spring. The change in the potential energy of the system can then be given as

$$
\begin{aligned}
\delta U & \triangleq-f_{e}^{T}\left(p_{A}-p_{B}\right)= \\
& =-K\left(y_{A}-y_{E}-d_{0}-d_{1}-d_{2}\right)\left[\begin{array}{ll}
0 & 1
\end{array}\right] \delta\left[\begin{array}{c}
x_{B} \\
y_{B}
\end{array}\right] \\
& =-K\left(y_{A}-y_{E}-d_{0}-d_{1}-d_{2}\right)
\end{aligned}
$$




$$
\begin{aligned}
& \times\left[\begin{array}{ll}
0 & 1
\end{array}\right] \delta\left[\begin{array}{c}
x_{B} \\
y_{E}+d_{1}+d_{2}
\end{array}\right] \\
= & -K\left[\left(y_{A}-d_{0}\right)-\left(y_{E}+d_{1}+d_{2}\right)\right] \\
& \times \delta\left(y_{E}+d_{1}+d_{2}\right) \\
= & \delta\left[\frac{1}{2} K\left[\left(y_{A}-d_{0}\right)-\left(y_{E}+d_{1}+d_{2}\right)\right]^{2}\right] .
\end{aligned}
$$

We remark that only the potential energy of the spring has been considered here because the center of mass of the follower is assumed to be placed at a fixed point. Consequently, the variation of the potential energy related to the gravity is assumed to be null.

Integrating Equation (5), we obtain an explicit expression for $U$ as

$U=\frac{1}{2} K\left[\left(y_{A}-d_{0}\right)-\left(y_{E}+d_{1}+d_{2}\right)\right]^{2}$.

Notice that, from Fig. 10, $d_{2}$ can be easily obtained as a function of the follower angular position $\theta_{f}$ and the parameter $d$ as

$d_{2}\left(\theta_{f}\right)=\frac{d}{\cos \left(\theta_{f}\right)}$

and, since any spring rotation is impossible by design, i.e. $x_{E}=x_{A}$, it is straightforward to write

$y_{E}\left(\theta_{f}\right)=x_{A} \tan \left(\theta_{f}\right)$.

Taking into account that $T=\frac{1}{2} J{\dot{\theta_{f}}}^{2}$ and substituting expressions (6) and (8) into Equation (4), we finally obtain

$$
\begin{aligned}
L= & \frac{1}{2} J{\dot{\theta_{f}}}^{2}-\frac{1}{2} K\left[\left(y_{A}-d_{0}\right)\right. \\
& \left.-\left(x_{A} \tan \left(\theta_{f}\right)+d_{1}+d_{2}\left(\theta_{f}\right)\right)\right]^{2} .
\end{aligned}
$$

\subsubsection{Unconstrained mode}

Considering expression (9), the mathematical description of the unconstrained motion of the follower can be simply obtained by solving Equation (3) in terms of the follower angular position $\theta_{f}$, thus yielding

$$
J \ddot{\theta_{f}}+K\left(x_{A} \tan \left(\theta_{f}\right)+\frac{d}{\cos \left(\theta_{f}\right)}-\left(y_{A}-d_{0}-d_{1}\right)\right)
$$

$$
\times\left(\frac{x_{A}}{\cos ^{2}\left(\theta_{f}\right)}+d \frac{\sin \left(\theta_{f}\right)}{\cos ^{2}\left(\theta_{f}\right)}\right)=0 .
$$

Notice that the right-end side of the above equation is zero since in the unconstrained mode only the conservative elastic force has to be taken into account.

\subsubsection{Constrained mode}

The contact mode equation can be obtained by treating the cam as an external input acting directly onto the follower.

Let $\hat{\theta}_{c}(t)$ be the angular position of the follower when the two bodies are in contact, then the torque $\tau$ provided by the cam has to be such that $\theta_{f}=\hat{\theta}_{c}$. Including the external forcing of the cam $\tau$ into Equation (10), we have the dynamical equation during permanent contact as:

$$
\begin{gathered}
J \ddot{\theta_{f}}+K\left(x_{A} \tan \left(\theta_{f}\right)+\frac{d}{\cos \left(\theta_{f}\right)}-\left(y_{A}-d_{0}-d_{1}\right)\right) \\
\quad \times\left(\frac{x_{A}}{\cos ^{2}\left(\theta_{f}\right)}+d \frac{\sin \left(\theta_{f}\right)}{\cos ^{2}\left(\theta_{f}\right)}\right)=\tau(t) .
\end{gathered}
$$

\subsection{Impacting law}

To model the transient contact between the follower and the cam, we need to add a collision rule to the system equations. Letting $t_{k}$ be the time instant when a generic impact occurs, such a rule gives the postimpact velocity, say $\dot{h}\left(t_{k}^{+}\right)$, as a function of the preimpact velocity $\dot{h}\left(t_{k}^{-}\right)$. In general, we have

$\dot{h}\left(t_{k}^{+}\right)=-r \dot{h}\left(t_{k}^{-}\right)$

where $r$ is the so-called coefficient of restitution [8].

In the case of interest, if the velocity of the contact point $p_{C}$ is continuous, we have

$\nabla \Sigma \cdot \dot{p_{F}}\left(t_{k}^{+}\right)=\nabla \Sigma \cdot \dot{p_{C}}\left(t_{k}\right)-r \dot{h}\left(t_{k}^{-}\right)$,

where $\Sigma$ is the lower side of the follower (assumed flat) oriented towards the cam (see Fig. 10). An analytical expression of $\Sigma$ as a function of $\theta_{f}$ is derived in Appendix A.

After simple algebraic manipulations (also reported in Appendix A), the impacting law can be finally 
expressed in terms of the follower angular position $\theta_{f}$ and velocity $\dot{\theta}_{f}$ as

$\dot{\theta_{f}}\left(t_{k}^{+}\right)=-r \dot{\theta_{f}}\left(t_{k}^{-}\right)+(1+r) \frac{\cos \left(\theta_{f}\right) \dot{y}_{c}-\sin \left(\theta_{f}\right) \dot{x_{c}}}{\cos \left(\theta_{f}\right) x_{c}+\sin \left(\theta_{f}\right) y_{c}}$.

Notice that Equation (14) is dependent upon the coordinates of the contact point $p_{C} \equiv\left(x_{c}, y_{c}\right)$ and their derivatives. Such coordinates are a function of the cam geometry, position and velocity. In the case of interest, the cam is assumed to have an eccentric circular shape. Therefore, as shown in Appendix B, we have

$\left\{\begin{array}{l}x_{c}\left(\theta_{c}\right)=e \cos \left(\theta_{c}\right)-R \sin \left(\hat{\theta}_{c}\left(\theta_{c}\right)\right)+x_{0} \\ y_{c}\left(\theta_{c}\right)=e \sin \left(\theta_{c}\right)+R \cos \left(\hat{\theta}_{c}\left(\theta_{c}\right)\right)+y_{0}\end{array}\right.$

and

$$
\left\{\begin{array}{l}
\dot{x}_{c}\left(\theta_{c}\right)=-e \sin \left(\theta_{c}\right) \dot{\theta}_{c}-R \cos \left(\hat{\theta}_{c}\left(\theta_{c}\right)\right) \frac{\partial \hat{\theta}_{c}}{\partial \theta_{c}}\left(\theta_{c}\right) \dot{\theta}_{c} \\
\dot{y}_{c}\left(\theta_{c}\right)=e \cos \left(\theta_{c}\right) \dot{\theta}_{c}-R \sin \left(\hat{\theta}_{c}\left(\theta_{c}\right)\right) \frac{\partial \hat{\theta}_{c}}{\partial \theta_{c}}\left(\theta_{c}\right) \dot{\theta}_{c}
\end{array}\right.
$$

where $R$ is the radius of the cam, $e$ is its eccentricity and $\hat{\theta}_{c}\left(\theta_{c}\right)$ is the follower angular position when there is contact between the two bodies. Also, as explained in Appendix B, we can obtain an analytical expression of $\hat{\theta}_{c}$, as:

$$
\begin{aligned}
& \hat{\theta}_{c}\left(\theta_{c}\right) \\
& =\arcsin \left(\frac{d+R}{\sqrt{\left(e \cos \left(\theta_{c}\right)+x_{0}\right)^{2}+\left(e \sin \left(\theta_{c}\right)+y_{0}\right)^{2}}}\right) \\
& \quad-\arctan \left(-\frac{e \sin \left(\theta_{c}\right)+y_{0}}{e \cos \left(\theta_{c}\right)+x_{0}}\right) .
\end{aligned}
$$

Note that the model derived above contains several non-linearities and can be ill-conditioned (high stiffness coefficient). These are well-known obstacles for numerical integration schemes that may require extremely small time steps and robust integrators. It is not the purpose of the present paper to discuss integration methods but we are completely aware of the difficulties resulting from both non-linearities and high frequencies. To overcome the numerical problems here, we used an event driven integration algorithm with adaptive stepsize implemented in the commercial platform MATLAB [26]. During simulations, when an impact occurs, the restitution law has been taken into account by a reset in the velocity follower value according to Equation (14).

\section{Identification of the model parameters}

In order to perform the numerical validation of the experimental results presented above, the next step is that of identifying the appropriate values of the model parameters. Many of the parameters can be obtained directly from the experiment as they are related to its physical and geometrical features. The only notable exception is the restitution coefficient $r$.

Table 5 contains all the values of the parameters which are needed to describe the follower motion, in the absence of impacts. The model is then validated against the experiment in Fig. 11 where the unconstrained follower motion is shown. As expected, the model shows a good agreement with the experimental data as highlighted from the time history of the error shown in Fig. 11(b).

\subsection{Estimating the coefficient of restitution}

The coefficient of restitution $r$ is an index of how elastic a collision is. The problem of estimating $r$ experimentally has been discussed in a large number of papers in the literature on impacting systems and it is usually based on bouncing ball experiments, for example, see $[1,45]$. The most common methods are usually based on high-speed data collection of the impact sounds as explained in [6], or on detailed analysis based on the use of high-speed cameras and force sensors as in [30]. The most basic approach is to consider $r$ as a constant

Table 5 Model parameters

\begin{tabular}{ll}
\hline Parameter & Value \\
\hline$R$ cam radius & $0.045 \mathrm{~m}$ \\
$e$ cam eccentricity & $0.015 \mathrm{~m}$ \\
$\left(x_{0}, y_{0}\right)$ center of rotation of the cam & $(0.249,0) \mathrm{m}$ \\
$d$ half of the follower height & $0.021 \mathrm{~m}$ \\
$J$ moment of inertia of the follower & $0.043 \mathrm{~kg} \mathrm{~m}$ \\
$K$ spring coefficient & $105 \mathrm{~N} / \mathrm{m}$ \\
$x_{A} x$-coordinate of the spring hooking point & $-0.031 \mathrm{~m}$ \\
$y_{A}-d_{0}-d_{1}$ spring elongation distance & $0.173 \mathrm{~m}$ \\
\hline
\end{tabular}




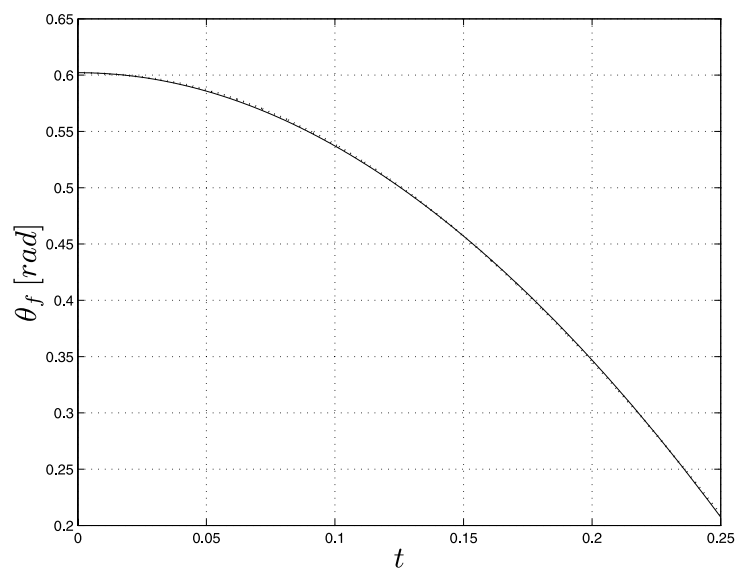

(a)

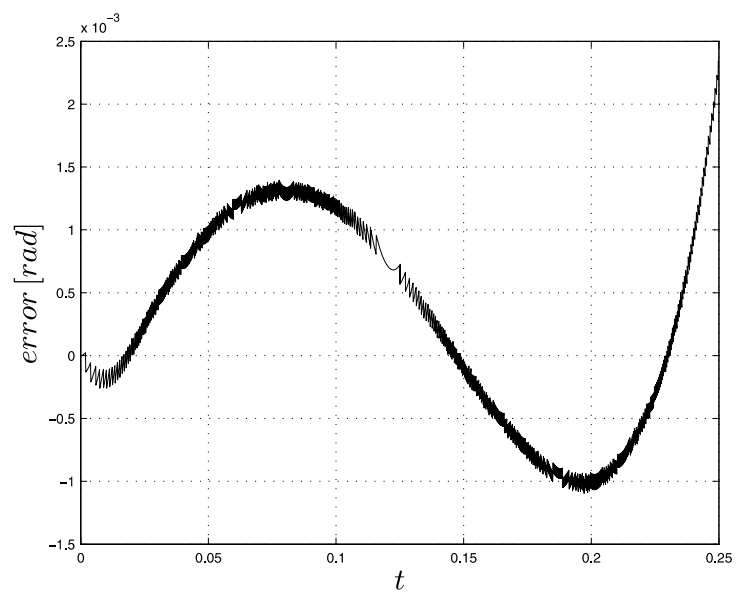

(b)

Fig. 11 Validation results. Time history of the follower free-fall motion: (a) $\theta_{f}(t)$ experimental (dashed) vs. numerical (solid); (b) estimation error corresponding to difference between data plotted in (a)

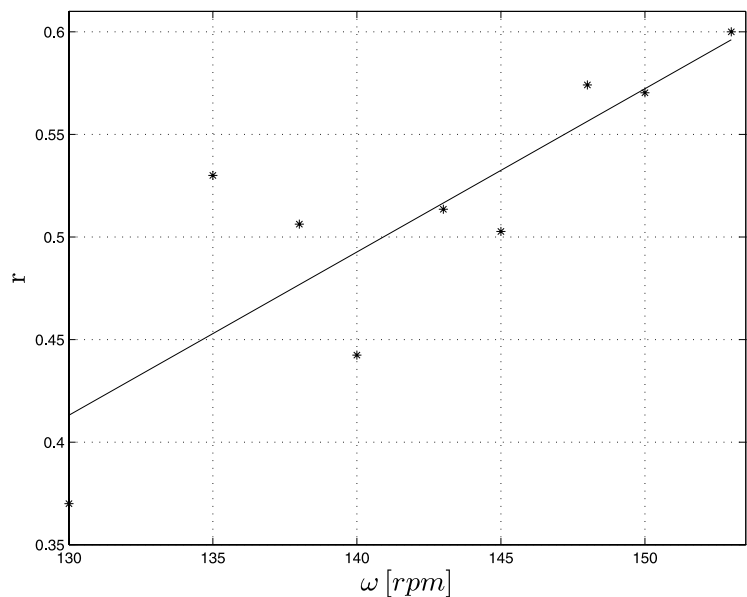

Fig. 12 Coefficient of restitution $r$ as a function of the cam velocity $\omega$ : experimental points (asterisks), linear approximation (solid line)

coefficient, whereas in practice it is well known that $r$ is actually an unknown function of the impact speed [6].

Since the impact velocity is not easy to measure, in this work, we assume that the coefficient of restitution $r$ is a function of the cam rotational speed. This is motivated by the observation that higher cam velocities lead to larger detachment of the follower and hence higher approach speed at the impact. In particular, we identified different values of $r$ by running a set of experiments at different constant values of $\omega$ ranging from 130 to $155 \mathrm{rpm}$. Given a fixed cam ve-

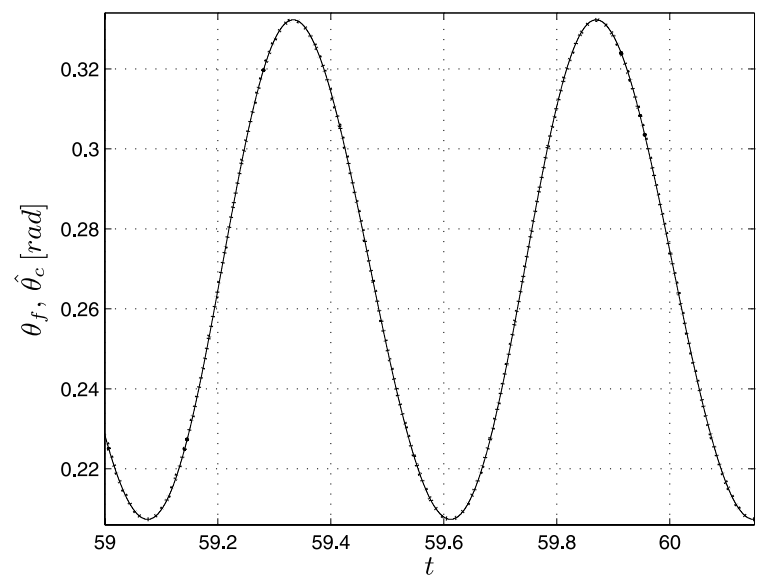

Fig. 13 Numerical time evolution of $\hat{\theta}_{c}$ (dotted line) and $\theta_{f}$ (solid line) for $\omega=110 \mathrm{rpm}$. This result agrees with waveform features of experimental low velocity range (see Fig. 3)

locity in the range of interest, $\omega \in[130,155] \mathrm{rpm}$, the coefficient $r$ is estimated from the observed first bouncing height. A least-square approach provides a linear interpolation of $r$ as a function of $\omega$. Results are shown in Fig. 12 where the solid line represents the linear least-squares fit which is used to obtain the numerical results presented in the rest of the paper. Although using the cam velocity instead of the actual approach speed in estimating $r$ is a strong approximation, the good agreement between simulations and experiments seems to confirm the validity of the adopted approach. 


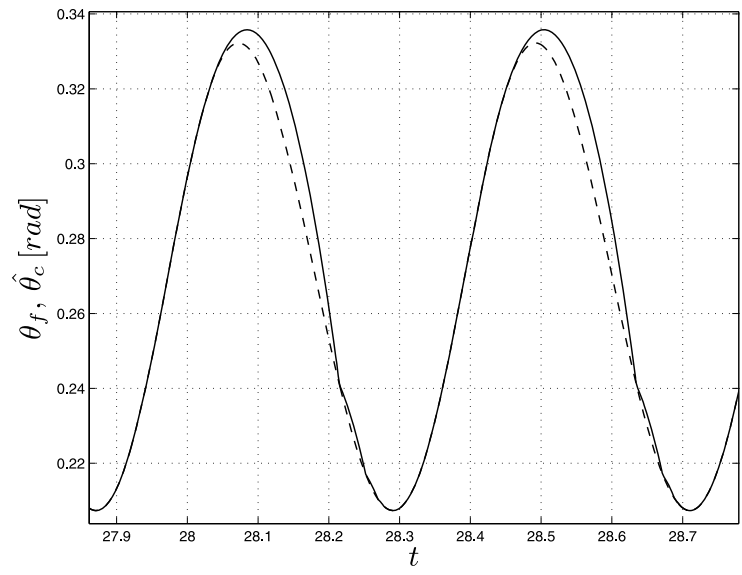

(a)

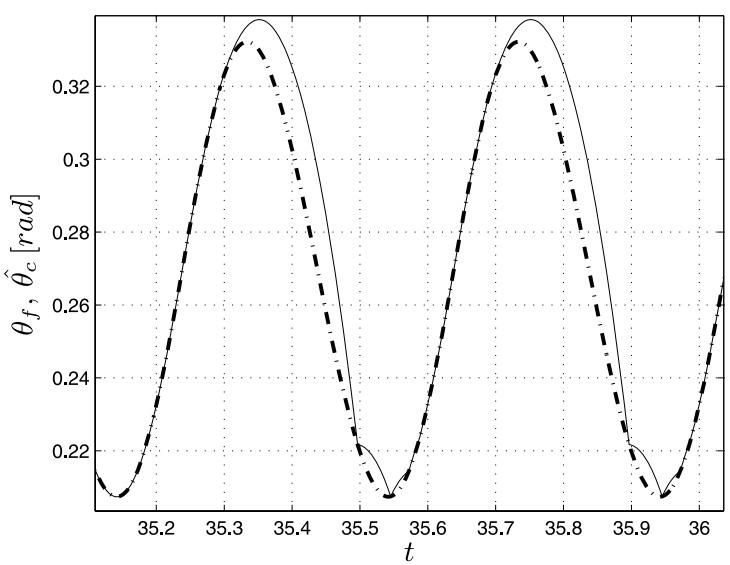

(c)

Fig. 14 Sections of numerical time series for the cam (dashed line) and follower (solid line) positions and their corresponding difference $\Delta \theta$ at: (a), (b) $\omega=143 \mathrm{rpm}$ and (c), (d) $\omega=150 \mathrm{rpm}$.

\subsection{Simulation results}

Using the model and the parameters obtained as discussed in the previous section, we perform a set of numerical simulations at the same values of the cam speed used in the experiments. We present the results of the simulation in the three regions associated to the different qualitative behaviour observed in the experiments.

\subsection{Permanent contact and detachment}

We start our numerical investigation by using low values of the cam rotational speed. As shown by contrasting Fig. 13 with Fig. 3, we obtained a remarkable agree-

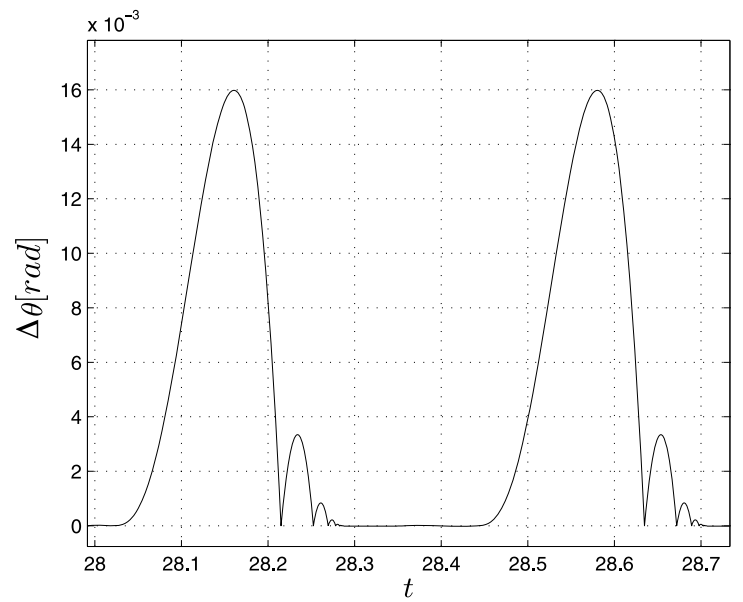

(b)

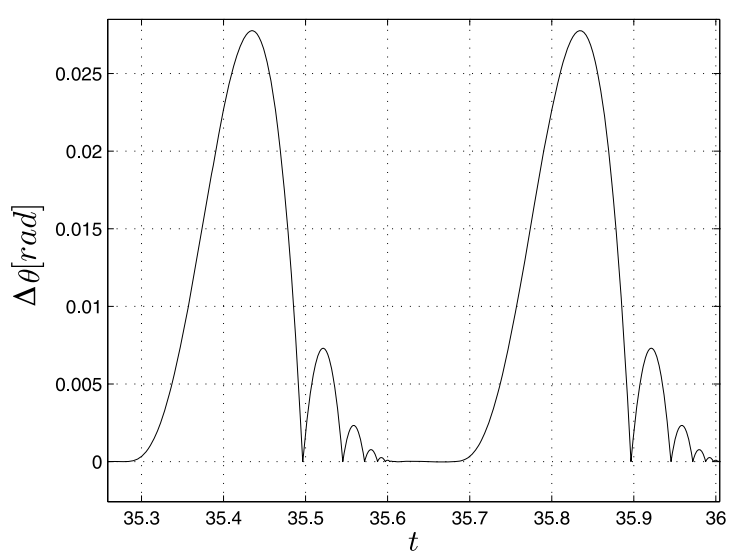

(d)

These results agrees with waveform features of experimental periodic regime (see Fig. 5)

ment between the experimental and numerical data confirming the presence of permanent contact in the low range of $\omega$ values.

By using the mathematical model, we were able to compute analytically the value of $\omega$ at which detachment between the cam and follower is bound to occur. By using an inverse kinematic approach [35], this value was computed to be $125 \mathrm{rpm}$ in perfect agreement with the experimental results reported above.

\subsection{Periodic regime}

When $\omega$ is increased past the critical value at which detachment occurs, the numerics show the same qual- 


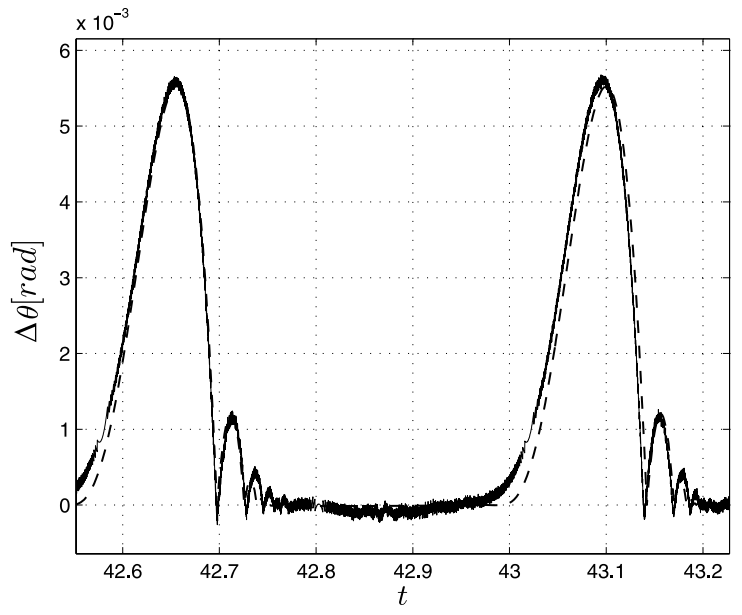

(a)

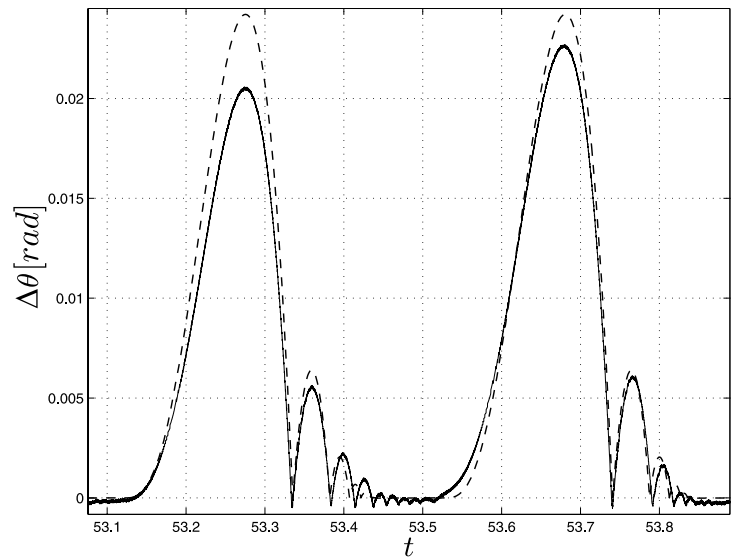

(c)

Fig. 15 Comparison between sections of simulated (dashed) and experimental (solid) differential $\Delta \theta$ time series for (a) $\omega=135 \mathrm{rpm}$, (b) $\omega=143 \mathrm{rpm}$, (c) $\omega=148 \mathrm{rpm}$ and (d) $\omega=$

itative behaviour observed experimentally. Namely, as shown in Figs. 14 and 15, we observe a good agreement between the periodic behaviour observed experimentally and the simulated trajectories. This also confirms the validity of the estimation strategy used to obtain the value of the coefficient of restitution $r$ as a function of the cam rotational speed $\omega$.

\subsection{Bifurcation diagram}

We can now verify whether the model captures the bifurcation behaviour observed experimentally in order to provide a further insight on whether the sudden transition to a seemingly aperiodic behaviour observed in the experiment is indeed a feature of the cam-follower system of interest. The numerical bifurcation diagram

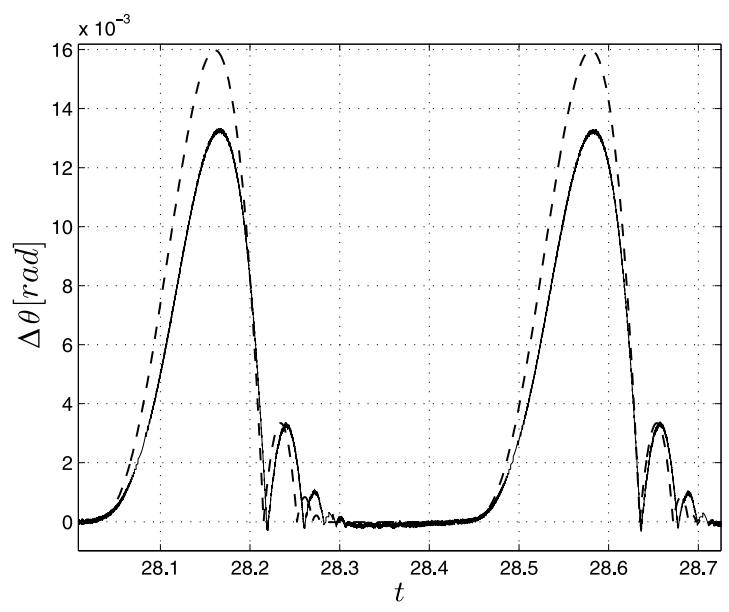

(b)

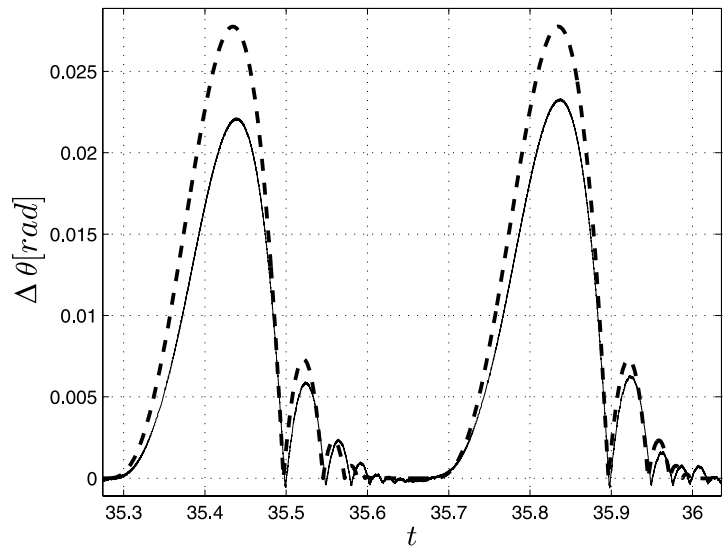

(d)

$150 \mathrm{rpm}$. This results evidence the preservation of dissipative features in the numerical approach from the first bounce

is shown in Fig. 16 and compared with the experimental one in Fig. 17. The agreement between the numerical and the experimental diagram is remarkable. This confirms that at a critical value of the cam rotational speed the system exhibits a sudden transition from the apparently robust periodic behaviour born after detachment to a seemingly aperiodic attractor.

The theoretical classification of this bifurcation is currently under investigation. Preliminary results indicate that the sudden transition detected both experimentally and numerically cannot be explained simply in terms of a grazing bifurcation typical of impacting systems. There are strong indications that such a transition might be due to the relative position of the impact point of the follower along the cam surface when an impact occurs. In particular, we conjecture 


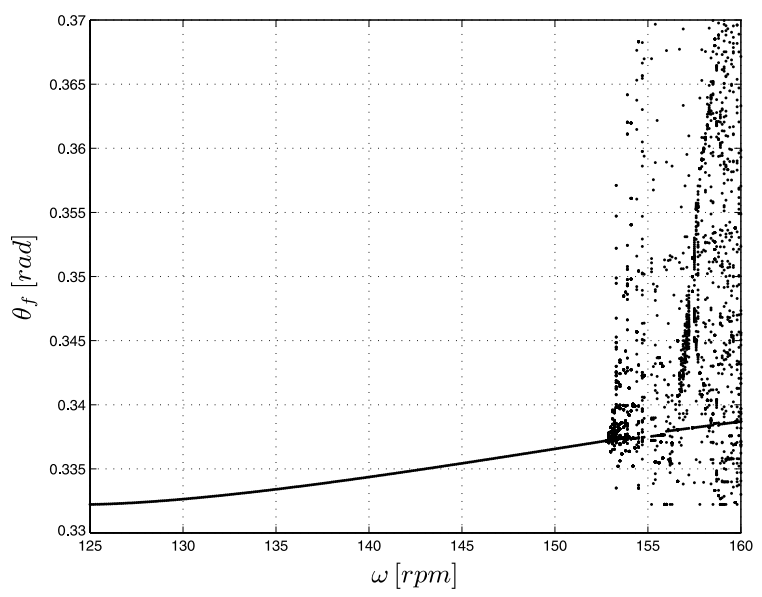

Fig. 16 Numerical bifurcation diagram
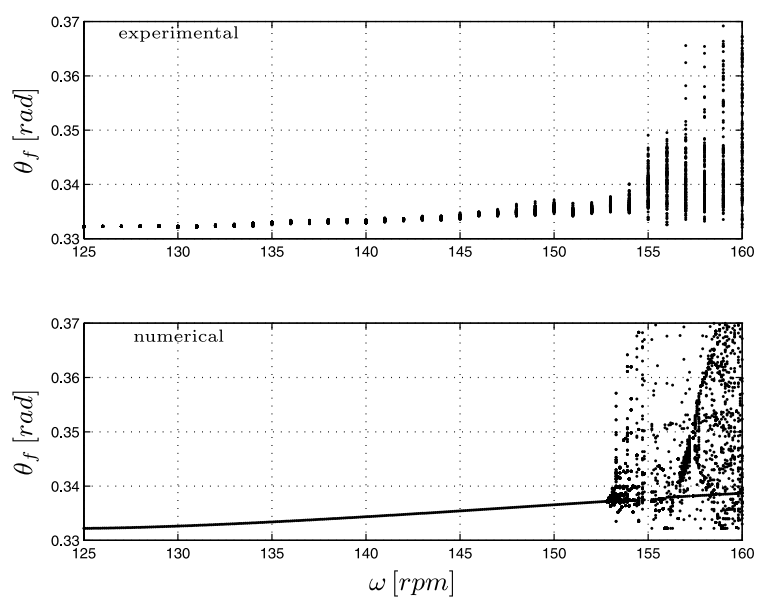

Fig. 17 Comparison between experimental and numerical bifurcation diagrams

that this phenomenon might be the smooth counterpart of the corner-impact bifurcation whose existence was recently conjectured in [11]. This is the subject of ongoing work.

\section{Conclusions}

We reported the design, modelling and analysis of a novel cam-follower mechanical rig to test and validate the complex behaviour of impacting mechanical systems. After presenting the main features of the proposed experimental apparatus, we carried out an extensive experimental investigation of its dynamics. We observed that the system can exhibit complex behaviour and aperiodic solutions following the detachment of the follower past a critical value of the cam rotational speed. A careful modelling of the rig was carried out and used to verify the experimental results. By using an appropriate strategy for the identification of the coefficient of restitution as a function of $\omega$, it was confirmed that the model of the rig captures the sudden transition from periodic to aperiodic solutions observed in the experiments. Preliminary results seem to show that such transition is due to a novel class of bifurcations which is currently under investigation.

We wish to emphasize that the results reported in this paper can be helpful: (i) to establish the cam-follower experimental rig as a versatile and flexible tool for the experimental analysis of bifurcations in impacting systems and (ii) to uncover the mechanisms leading to the occurrence of complex behaviour in such systems. For example, as suggested in [11,38], different cam profiles can be easily mounted on the experiment in order to validate the theoretical results on bifurcations of impacting systems. Also, gaining a better knowledge of the complex behaviour associated to the detachment of the follower from the cam can support the design of more sophisticated controllers aiming at avoiding its occurrence.

\section{Appendix A: Derivation of Equation (14)}

We report here some of the analytical derivations required to obtain the model presented in Section 4. The impacting law given in (14) can be obtained by recasting the quantities in (13) as functions of the follower angular position and velocity together with the coordinates of the contact point $p_{C}$.

The first step is to express $h$ and $\nabla \Sigma \cdot \dot{p_{C}}$ in terms of the coordinates of the generic contact point, $\left(x_{c}\right.$, $y_{c}$ ), and the follower angular position $\theta_{f}$. From the geometry depicted in Fig. 10, $h$ is the distance between the straight line of slope $\tan \left(\theta_{f}\right)$ passing through the point $(0,-\bar{d})$ in the $(x, y)$ coordinate system and the point on the cam $p_{C}=\left(x_{c}, y_{c}\right)$. Then, simple geometric arguments yield the following expression for $h$ :

$h\left(x_{c}, y_{c}, \theta_{f}\right)=\sin \left(\theta_{f}\right) x_{c}-\cos \left(\theta_{f}\right) y_{c}-d$.

Differentiating with respect to time (18), we have

$$
\begin{aligned}
\dot{h}\left(x_{c}, y_{c}, \theta_{f}\right)= & \sin \left(\theta_{f}\right) \dot{x}_{c}-\cos \left(\theta_{f}\right) \dot{y}_{c}+\left(\cos \left(\theta_{f}\right) x_{c}\right. \\
& \left.+\sin \left(\theta_{f}\right) y_{c}\right) \dot{\theta_{f}} .
\end{aligned}
$$


Analogously, it is also possible to express $\Sigma$ in the $(x, y)$ coordinate system. Namely, from Fig. 10, it follows that

$\Sigma:=\left\{(x, y): y=\tan \left(\theta_{f}\right) x-\frac{d}{\cos \left(\theta_{f}\right)}\right\}$

and then, since we can define $\nabla \Sigma=\left[-\sin \left(\theta_{f}\right)\right.$ $\left.\cos \left(\theta_{f}\right)\right]$, we obtain

$\nabla \Sigma \cdot \dot{p}_{C}=-\sin \left(\theta_{f}\right) \dot{x}_{c}+\cos \left(\theta_{f}\right) \dot{y}_{c}$.

The second step is the derivation of an expression for $\nabla \Sigma \cdot \dot{p}_{F}$ in terms of $\dot{\theta}_{f}$. Note that $p_{F}$ has coordinates $\left(x_{f}, y_{f}\right)$ related to $(l,-d)$ by the rotation matrix $\Xi\left(\theta_{f}\right)$ defined as

$\Xi\left(\theta_{f}\right)=\left[\begin{array}{cc}\cos \left(\theta_{f}\right) & -\sin \left(\theta_{f}\right) \\ \sin \left(\theta_{f}\right) & \cos \left(\theta_{f}\right)\end{array}\right]$.

Simple algebraic manipulations then yield:

$$
\begin{aligned}
\nabla \Sigma \cdot \dot{p_{F}}= & {\left[-\sin \left(\theta_{f}\right) \cos \left(\theta_{f}\right)\right] } \\
& \times\left[\begin{array}{l}
-\sin \left(\theta_{f}\right) l+\cos \left(\theta_{f}\right) d \\
\cos \left(\theta_{f}\right) l+\sin \left(\theta_{f}\right) d
\end{array}\right] \dot{\theta_{f}}=l \dot{\theta_{f}} .
\end{aligned}
$$

When an impact occurs, we also have $p_{F} \equiv p_{C}$, and therefore

$l=\hat{t}^{T}\left[\begin{array}{l}x_{c} \\ y_{c}\end{array}\right]=\cos \left(\theta_{f}\right) x_{c}+\sin \left(\theta_{f}\right) y_{c}$.

Finally, expression (14) in Section 4.2 can be obtained by substituting (19), (21) and (23) into Equation (13).

\section{Appendix B: Derivation of Equations (15)-(17)}

The coordinates of the contact point $p_{C}=\left(x_{c}, y_{c}\right)$, can be obtained in terms of the cam rotational angle $\theta_{c}$ for a cam with an eccentric circular shape as follows.

Firstly, we need to derive again an expression for the distance between the cam and the follower, $h$. This can be obtained by subtracting the radius $R$ of the cam from the distance between the cam geometric center, say $p_{G}=\left(x_{g}, y_{g}\right)$, and the point $p_{F}$ on the follower (see
Fig. 10). The coordinates of the point $p_{G}$ can be obtained in terms of the coordinates of the cam rotational center, $\left(x_{0}, y_{0}\right)$, by simple trigonometric considerations as:

$\left\{\begin{array}{l}x_{g}\left(\theta_{c}\right)=e \cos \left(\theta_{c}\right)+x_{0}, \\ y_{g}\left(\theta_{c}\right)=e \sin \left(\theta_{c}\right)+y_{0} .\end{array}\right.$

Then, straightforward algebraic manipulations give:

$$
\begin{aligned}
h\left(\theta_{f}, \theta_{c}\right)= & {\left[e \sin \left(\theta_{c}\right)+x_{0}\right] \sin \left(\theta_{f}\right) } \\
& -\left[e \sin \left(\theta_{c}\right)+y_{0}\right] \cos \left(\theta_{f}\right)-d-R .
\end{aligned}
$$

Note that, for a generic $\theta_{c}$, it is possible to solve the trigonometric equation $h\left(\theta_{f}, \theta_{c}\right)=0$ which gives analytically the angular displacement at the follower joint when the cam and the follower are permanently in contact. This is precisely the expression given in (17).

Now, let $\hat{n}$ be the unit vector lying along the direction of the lower side of the follower in contact with the cam. Clearly, $\hat{n}$ is given by:

$\hat{n}\left(\hat{\theta}_{c}\left(\theta_{c}\right)\right)=\left[-\sin \left(\hat{\theta}_{c}\left(\theta_{c}\right)\right) \cos \left(\hat{\theta}_{c}\left(\theta_{c}\right)\right)\right]^{T}$.

Then, (15) can be obtained by adding to the coordinates of the cam geometrical center, $p_{G}$, given by (25), the vector having modulus equal to $R$ and direction orthogonal to $\hat{n}$. Finally, differentiating (15), we obtain (16).

Acknowledgements The authors would like to acknowledge support from the European Union V Framework Programme Project SICONOS IST2001-37172 and the Italian Ministry of Research and University Project PRIN 2005 (MACSI) on the analysis and control of hybrid systems. Also, we would like to thank the anonymous reviewers for their insightful comments which greatly improved the manuscript and its clarity. Also, we are grateful to Dr. Petri Piiroinen (University of Bristol) for all the useful comments and discussions and the M.Sc. students Luca Avitabile and Armando Vollono from University of Naples - Federico II, who actively contributed to the design and validation of the experimental rig during their M.Sc. thesis work.

\section{References}

1. Aguiar, C.E., Laudares, F.: Listening to the coefficient of restitution and the gravitational acceleration of a bouncing ball. Am. J. Phys. 71, 499-501 (2003) 
2. Akiba, K., Sakai, H.: A comprehensive simulation of high speed driven valve trains. SAE Transactions - Society for Automotive Engineers (1981)

3. Azejczyk, B., Kapitaniak, T., Wojewoda, J., Barron, R.: Experimental-observation of intermittent chaos in a mechanical system with impacts. J. Sound Vib. 178, 272-275 (1994)

4. Barkan, P.: Calculation of high speed valve motion with a flexible overhead linkage. SAE Transactions - Society for Automotive Engineers 61, 687-700 (1953)

5. Bayly, P.V., Virgin, L.N.: An experimental study of an impacting pendulum. J. Sound Vib. 164(2), 364-374 (1993)

6. Bernstein, A.: Listening to the coefficient of restitution. Am. J. Phys. 45, 41-44 (1977)

7. Blazejczyk-Okolewska, B., Czolczynski, K., Kapitaniak, J., Wojewoda, T.: Chaotic Mechanics in Systems with Impacts and Friction. World Scientific, Singapore (1999)

8. Brogliato, B.: Nonsmooth Mechanics. Springer, London (1999a)

9. Brogliato, B.: Nonsmooth Mechanics - Models, Dynamics and Control. Springer-Verlag, New York (1999b)

10. Brooker, G.: Development of a W-band scanning conscan antenna based on thetwist-reflector concept. In: Proceedings of the ICMMT - 2nd International conference on Microwave and Millimeter Wave Technology, Sydney, NSW, pp. 436439 (2000)

11. Budd, C., Piiroinen, P.: Corner bifurcations in non-smoothly forced impact oscillators. Physica D 220, 127-145 (2006)

12. Carlini, A., Rivola, A., Dalpiaz, G., Maggiore, A.: Valve motion measurements on motorbike cylinder heads using high speed laser vibrometer. In: Proceedings of the Conference on Vibration Measurements by Laser Techniques, Ancona, Italy (2002)

13. Caton, J.A.: Operating characterising of a spark-ignition engine using the second law of thermodynamics: effect of speed and load. SAE Transactions, Paper 2000-01-0952, Detroit, MI (2000)

14. Chae, A., Hollerbach, J.: Dynamic stability issues in force control of manipulators. Proc. IEEE Int. Conf. Robot. Autom. 4, 890-896 (1987)

15. Choi, T.D., Eslinger, O.J., Kelley, C.T., David, J.W., Etheridge, M.: Optimization of automotive valve train components with implicit filtering. Optim. Eng. 1(1), 9-27 (2000)

16. Demeulenaere, B., Schutter, J.D.: Dynamically compensated cams for rigid cam-follower systems with fluctuating cam speed and dominating inertial forces. In: IEEE/ASME International Conference on Advanced Intelligent Mechatronics, Como, Italy, pp. 763-767 (2001)

17. di Bernardo, M., Alzate, R., Santini, S.: Deliverable wp6. D6.4: experimental Validation of non-smooth Bifurcations. Part III. Technical report, SICONOS Project - University of Naples (2006)

18. di Bernardo, M., Budd, C., Champneys, A., Kowalczyk, P., Nordmark, A., Olivar, G., Piiroinen, P.: Bifurcations in nonsmooth dynamical systems. SIAM - Society for Industrial and Applied Mathematics. Review (2006)

19. di Bernardo, M., Budd, C.J., Champneys, A.R., Kowalczyk, P.: Bifurcation and Chaos in Piecewise Smooth Dynamical Systems - Theory and Applications. Springer-Verlag, UK (2007)

20. Dickrell, D.J., Dooner, D.B., Sawyer, W.G.: The evolution of geometry for a wearing circular cam: analytical and com- puter simulation with comparison to experiment. J. Tribol. 125, 187-192 (2003)

21. Dresner, T.L., Barkan, P.: New methods for the dynamical analysis of flexible single-input and multi-input camfollower systems. J. Mech. Des. 17, 151 (1995)

22. Hamidzadeh, H.R., Dehghani, M.: Dynamic stability of flexible cam follower systems. Proc. Inst. Mech. Eng., Part K: J. Multibody Dyn. 213(1), 45-52 (1999)

23. Hengstler: Incremental Shaft Encoders: Type RI 58. Technical report, HENGSTLER, http://www.hengstler.de/ (2001)

24. Hengstler: DS1104 R\&D Controller Board: Features. Technical report, dSPACE Inc., http://www.dspaceinc.com/ww/en/inc/home.cfm (2005)

25. Heywood, J.: Internal Combustion Engine Fundamentals. McGraw-Hill, New York (1998)

26. Higham, D.J., Higham, N.J.: Matlab Guide. Society for Industrial and Applied Mathematics, Philadelphia (2000)

27. Hinrichs, N., Oestreich, M., Popp, K.: Experiments, modelling and analysis of friction and impact oscillators. Zeitschrift fur angewandte mathematik und mechanik 79, S95-S96 (1999)

28. Koster, M.P.: Vibrations of Cam Mechanisms. Phillips Technical Library Series, Macmillan Press Ltd., London (1974)

29. Kushwaha, M., Rahnejat, H.: Valve-train dynamics: a simplified tribo-elasto-multi-body analysis. Proc. Inst. Mech. Eng., Part K: J. Multibody Dyn. 214, 1464-4193 (2001)

30. Labous, L., Rosato, A., Dave, R.N.: Measurement of collisional properties of spheres using high-speed video analysis. Phys. Rev. E 56, 5717-5725 (1997)

31. Leine, R., Nijmeijer, H.: Dynamics and Bifurcations in NonSmooth Mechanical Systems. Springer-Verlag (2004)

32. Liou, J.J., Huang, G., Hsu, W.: Experimental study of a variable pressure damper on an automotive valve train. J. Mech. Des. 120(2), 279-281 (1998)

33. Mosekilde, E., Zhusubalyev, Z.: Bifurcations and Chaos in Piecewise-Smooth Dynamical Systems. World Scientific, Singapore (2003)

34. Nordmark, A.B.: Non-periodic motion caused by grazing incidence in impact oscillators. J. Sound Vib. 2, 279-297 (1991)

35. Norton, R.: Cam Design and Manufacturing Handbook. Industrial-Press, New York (2002)

36. Norton, R., Eovaldi, J., Westbrook, D., Stene, R.L.: Effect of the valve-cam ramps on valve train dynamics. SAE Transactions - Society for Automotive Engineers, Paper 1999-010801 (1999)

37. Oestreich, M., Hinrichs, N., Popp, K., Budd, C.J.: Analytical and experimental investigation of an impact oscillator. In: Proceedings of the ASME - 16th Biennal Conference on Mechanical Vibrations and Noise DETC97VIB-3907, pp. 1-11 (1997)

38. Osorio, G., Di Bernardo, M., Santini, S.: Chattering and complex behavior of a cam-follower system. In: Proceedings of the European Nonlinear Dynamics Conference (ENOC). Eindhoven, The Netherlands (2005)

39. Piiroinen, P.T., Virgin, L.N., Champneys, A.R.: Chaos and period-adding; experimental and numerical verification of the grazing bifurcation. J. Nonlinear Sci. 14, 383-404 (2004)

40. Raghavacharyulu, E., Rao, J.: Jump phenomena in camfollower systems a continuous-mass-model approach. In: 
Proceedings of the Winter Annual Meeting, ASME, New York, NY pp. 1-8 (1976)

41. Rothbart, H.A.: Cams-Design, Dynamics, and Accuracy. Wiley, New York (1956)

42. Sanyodenki: SANMOTION Q: AC Servo Systems. Technical report, Sanyo-Denki Ltd., http://www.sanyo-denki.com/ (2002)

43. Seidlitz, S.: Valve train dynamics - a computer study. SAE Transactions - Society for Automotive Engineers, SAE Paper 890620 (1989)

44. Sherman, R.H., Blumberg, P.N.: The influence of induction and exhaust processes on emissions and fuel consumption in the spark ignited engine. SAE Transactions - Society for Automotive Engineers, Paper 770990 (1997)

45. Stensgaard, I., Laegsgaard, E.: Listening to the coefficient of restitution - revisited. Am. J. Phys. 69, 301-305 (2001)

46. Stensson, A., Nordmark, A.: Experimental investigation of some consequences of low velocity impacts in the chaotic dynamics of a mechanical system. Philosophical transactions: physical sciences and engineering. Roy. Soc. Lond. A 347, 439-448 (1994)

47. Teodorescu, M., Votsios, V., Rahnejat, H., Taraza, D.: Jounce and impact in cam-tappet conjunction induced by the elasto- dynamics of valve train system. Meccanica 41(2), 157-171 (2006)

48. Todd, M.D., Virgin, L.N.: An experimental impact oscillator. Chaos Solitons Fractals 8, 699-714 (1997)

49. Tumer, T.S., Unlusoy, Y.S.: Nondimensional analysis of jump phenomenon in force-closed cam mechanisms. Mech. Mach. Theory 6, 421-432 (1991)

50. Urin, A.M.: Experience with the use of PK-profile joints in agricultural machines. Research, design, calculations, and experience of operation of chemical equipment technology. Chem. Pet. Eng. 34(8), 534-536 (1998)

51. Wiercigroch, M., de Kraker, B.: Applied Nonlinear Dynamics and Chaos of Mechanical Systems with Discontinuities. World Scientific Publishing, Singapore (2000)

52. Wiercigroch, M., Pavlovskaia, E.: Nonlinear dynamics of vibro-impact systems: Theory and experiments. Mod. Pract. Stress Vib. Anal. 440-4, 513-520 (2003)

53. Yan, H.S., Tsai, M., Hsu, M.H.: An experimental study of the effect of the cam speed on cam-follower systems. Mech. Mach. Theory 31, 3971-412 (1996)

54. Zang, W., Ye, M.: Local and global bifurcations of valve mechanism. Nonlinear Dyn. 6, 301-316 (1994) 